Louisiana State University

LSU Digital Commons

$12-20-2019$

\title{
Evidence for Late-stage Eruptive Mass Loss in the Progenitor to SN2018gep, a Broad-lined Ic Supernova: Pre-explosion Emission and a Rapidly Rising Luminous Transient
}

\author{
Anna Y.Q. Ho \\ California Institute of Technology \\ Daniel A. Goldstein \\ California Institute of Technology \\ Steve Schulze \\ Weizmann Institute of Science Israel \\ David K. Khatami \\ University of California, Berkeley \\ Daniel A. Perley \\ Liverpool John Moores University
}

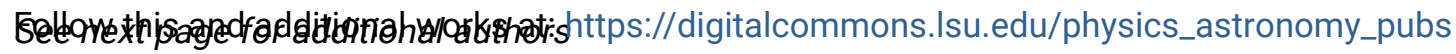

\author{
Recommended Citation \\ Ho, A., Goldstein, D., Schulze, S., Khatami, D., Perley, D., Ergon, M., Gal-Yam, A., Corsi, A., Andreoni, I., \\ Barbarino, C., Bellm, E., Blagorodnova, N., Bright, J., Burns, E., Cenko, S., Cunningham, V., De, K., Dekany, R., \\ Dugas, A., Fender, R., Fransson, C., Fremling, C., Goldstein, A., Graham, M., Hale, D., Horesh, A., Hung, T., \\ Kasliwal, M., M. Kuin, N., Kulkarni, S., Kupfer, T., Lunnan, R., \& Masci, F. (2019). Evidence for Late-stage \\ Eruptive Mass Loss in the Progenitor to SN2018gep, a Broad-lined Ic Supernova: Pre-explosion Emission \\ and a Rapidly Rising Luminous Transient. Astrophysical Journal, 887 (2) https://doi.org/10.3847/ \\ $1538-4357 / a b 55 e c$
}

This Article is brought to you for free and open access by the Department of Physics \& Astronomy at LSU Digital Commons. It has been accepted for inclusion in Faculty Publications by an authorized administrator of LSU Digital Commons. For more information, please contact ir@lsu.edu. 


\section{Authors}

Anna Y.Q. Ho, Daniel A. Goldstein, Steve Schulze, David K. Khatami, Daniel A. Perley, Mattias Ergon, Avishay Gal-Yam, Alessandra Corsi, Igor Andreoni, Cristina Barbarino, Eric C. Bellm, Nadia Blagorodnova, Joe S. Bright, E. Burns, S. Bradley Cenko, Virginia Cunningham, Kishalay De, Richard Dekany, Alison Dugas, Rob P. Fender, Claes Fransson, Christoffer Fremling, Adam Goldstein, Matthew J. Graham, David Hale, Assaf Horesh, Tiara Hung, Mansi M. Kasliwal, N. Paul M. Kuin, S. R. Kulkarni, Thomas Kupfer, Ragnhild Lunnan, and Frank J. Masci 


\section{PDF hosted at the Radboud Repository of the Radboud University Nijmegen}

The following full text is a publisher's version.

For additional information about this publication click this link.

https://hdl.handle.net/2066/215850

Please be advised that this information was generated on 2021-10-13 and may be subject to change. 


\title{
Evidence for Late-stage Eruptive Mass Loss in the Progenitor to SN2018gep, a Broad- lined Ic Supernova: Pre-explosion Emission and a Rapidly Rising Luminous Transient
}

Anna Y. Q. Ho ${ }^{1}$ (D), Daniel A. Goldstein ${ }^{1,27}$ (D), Steve Schulze ${ }^{2}$ (D), David K. Khatami ${ }^{3}$ (D), Daniel A. Perley ${ }^{4}$ (D), Mattias Ergon ${ }^{5}$, Avishay Gal-Yam ${ }^{2}$ (D), Alessandra Corsi ${ }^{6}$ (D) Igor Andreoni $^{1}$ (D), Cristina Barbarino ${ }^{5}$, Eric C. Bellm ${ }^{7}$ (D), Nadia Blagorodnova ${ }^{8}$ (i), Joe S. Bright ${ }^{9}$ (D) E. Burns ${ }^{10}$, S. Bradley Cenko ${ }^{11,12}$ (D), Virginia Cunningham ${ }^{13}$ (D), Kishalay De ${ }^{1}$ (iD), Richard Dekany ${ }^{14}$, Alison Dugas ${ }^{1}$, Rob P. Fender ${ }^{9}$, Claes Fransson ${ }^{5}$ (D), Christoffer Fremling ${ }^{15}$ (D), Adam Goldstein ${ }^{16}$ (D), Matthew J. Graham ${ }^{15}$ (D), David Hale $^{14}$, Assaf Horesh ${ }^{17}$, Tiara Hung ${ }^{18}$, Mansi M. Kasliwal ${ }^{1}$ (D), N. Paul M. Kuin ${ }^{19}$, S. R. Kulkarni ${ }^{1}$ (D), Thomas Kupfer ${ }^{20}$ (iD, Ragnhild Lunnan $^{5}$ (D), Frank J. Masci ${ }^{21}$ (D), Chow-Choong Ngeow ${ }^{22}$ (D), Peter E. Nugent ${ }^{23}$ (D), Eran O. Ofek ${ }^{2}$ (D),

Maria T. Patterson ${ }^{7}$ (D), Glen Petitpas ${ }^{24}$, Ben Rusholme ${ }^{21}$, Hanna Sai ${ }^{25}$, Itai Sfaradi ${ }^{17}$, David L. Shupe ${ }^{21}$, Jesper Sollerman ${ }^{5}$ (D), Maayane T. Soumagnac ${ }^{2}$ (D), Yutaro Tachibana ${ }^{26}$, Francesco Taddia ${ }^{5}$, Richard Walters ${ }^{1}$, Xiaofeng Wang ${ }^{25}$ (D), Yuhan Yao ${ }^{1}$ (D), and Xinhan Zhang 25

${ }^{1}$ Cahill Center for Astrophysics, California Institute of Technology, MC 249-17, 1200 E California Boulevard, Pasadena, CA 91125, USA

${ }^{2}$ Department of Particle Physics and Astrophysics, Weizmann Institute of Science, 234 Herzl Street, 76100 Rehovot, Israel

${ }^{3}$ Department of Astronomy, University of California, Berkeley, CA 94720, USA

${ }^{4}$ Astrophysics Research Institute, Liverpool John Moores University, IC2, Liverpool Science Park, 146 Brownlow Hill, Liverpool L3 5RF, UK

5 The Oskar Klein Centre \& Department of Astronomy, Stockholm University, AlbaNova, SE-106 91 Stockholm, Sweden

${ }^{6}$ Department of Physics and Astronomy, Texas Tech University, Box 1051, Lubbock, TX 79409-1051, USA

${ }^{7}$ DIRAC Institute, Department of Astronomy, University of Washington, 3910 15th Avenue NE, Seattle, WA 98195, USA

${ }^{8}$ Department of Astrophysics/IMAPP, Radboud University, Nijmegen, The Netherlands

${ }^{9}$ Department of Physics, University of Oxford, Denys Wilkinson Building, Keble Road, Oxford OX1 3RH, UK

${ }^{10}$ NASA Postdoctoral Program Fellow, Goddard Space Flight Center, Greenbelt, MD 20771, USA

${ }^{11}$ Astrophysics Science Division, NASA Goddard Space Flight Center, Mail Code 661, Greenbelt, MD 20771, USA

${ }^{12}$ Joint Space-Science Institute, University of Maryland, College Park, MD 20742, USA

${ }^{13}$ Astronomy Department, University of Maryland, College Park, MD 20742, USA

${ }^{14}$ Caltech Optical Observatories, California Institute of Technology, MC 11-17, 1200 E. California Boulevard, Pasadena, CA 91125, USA

${ }^{15}$ Division of Physics, Mathematics and Astronomy, California Institute of Technology, Pasadena, CA 91125, USA

${ }^{16}$ Science and Technology Institute, Universities Space Research Association, Huntsville, AL 35805, USA

${ }^{17}$ Racah Institute of Physics, Hebrew University, Jerusalem 91904, Israel

${ }^{18}$ Department of Astronomy and Astrophysics, University of California, Santa Cruz, CA 95064, USA

${ }^{19}$ Mullard Space Science Laboratory, University College London, Holmbury St. Mary, Dorking, Surrey RH5 6NT, UK

${ }^{20}$ Kavli Institute for Theoretical Physics, University of California, Santa Barbara, CA 93106, USA

${ }^{21}$ IPAC, California Institute of Technology, 1200 E. California Boulevard, Pasadena, CA 91125, USA

${ }^{22}$ Graduate Institute of Astronomy, National Central University, 32001, Taiwan

${ }^{23}$ Lawrence Berkeley National Laboratory, 1 Cyclotron Road, Berkeley, CA 94720, USA

${ }^{24}$ Harvard-Smithsonian Center for Astrophysics, 60 Garden Street, Cambridge, MA 02138, USA

${ }^{25}$ Physics Department/Tsinghua Center for Astrophysics, Tsinghua University; Beijing, 100084, People's Republic of China

${ }^{26}$ Department of Physics, Tokyo Institute of Technology, 2-12-1 Ookayama, Meguro-ku, Tokyo 152-8551, Japan

Received 2019 April 24; revised 2019 September 17; accepted 2019 October 1; published 2019 December 18

\begin{abstract}
We present detailed observations of ZTF18abukavn (SN2018gep), discovered in high-cadence data from the Zwicky Transient Facility as a rapidly rising $\left(1.4 \pm 0.1 \mathrm{mag} \mathrm{hr}^{-1}\right)$ and luminous $\left(M_{g \text {,peak }}=-20 \mathrm{mag}\right)$ transient. It is spectroscopically classified as a broad-lined stripped-envelope supernova (Ic-BL SN). The high peak luminosity $\left(L_{\text {bol }} \gtrsim 3 \times 10^{44} \mathrm{erg} \mathrm{s}^{-1}\right)$, the short rise time $\left(t_{\text {rise }}=3\right.$ days in $g$ band), and the blue colors at peak $(g-r \sim-0.4)$ all resemble the high-redshift Ic-BL iPTF16asu, as well as several other unclassified fast transients. The early discovery of SN2018gep (within an hour of shock breakout) enabled an intensive spectroscopic campaign, including the highest-temperature $\left(T_{\text {eff }} \gtrsim 40,000 \mathrm{~K}\right)$ spectra of a stripped-envelope SN. A retrospective search revealed luminous $\left(M_{g} \sim M_{r} \approx-14 \mathrm{mag}\right)$ emission in the days to weeks before explosion, the first definitive detection of precursor emission for a Ic-BL. We find a limit on the isotropic gamma-ray energy release $E_{\gamma, \text { iso }}<4.9 \times 10^{48} \mathrm{erg}$, a limit on X-ray emission $L_{\mathrm{X}}<10^{40} \mathrm{erg} \mathrm{s}^{-1}$, and a limit on radio emission $\nu L_{\nu} \lesssim 10^{37} \mathrm{erg} \mathrm{s}^{-1}$. Taken together, we find that the early $(<10$ days $)$ data are best explained by shock breakout in a massive shell of dense circumstellar material $\left(0.02 M_{\odot}\right)$ at large radii $\left(3 \times 10^{14} \mathrm{~cm}\right)$ that was ejected in eruptive pre-explosion mass-loss episodes. The late-time ( $>10$ days) light curve requires an additional energy source, which could be the radioactive decay of Ni-56.
\end{abstract}

Key words: methods: observational - shock waves - stars: mass-loss - supernovae: individual - surveys

Supporting material: machine-readable tables

${ }^{27}$ Hubble Fellow. 


\section{Introduction}

Recent discoveries by optical time-domain surveys challenge our understanding of how energy is deposited and transported in stellar explosions (Kasen 2017). For example, over 50 transients have been discovered with rise times and peak luminosities too rapid and too high, respectively, to be explained by radioactive decay (Poznanski et al. 2010; Drout et al. 2014; Arcavi et al. 2016; Shivvers et al. 2016; Tanaka et al. 2016; Pursiainen et al. 2018; Rest et al. 2018). Possible powering mechanisms include interaction with extended circumstellar material (CSM; Chevalier \& Irwin 2011), and energy injection from a long-lived central engine (Kasen \& Bildsten 2010; Woosley 2010; Kasen et al. 2016). These models have been difficult to test because the majority of fast-luminous transients have been discovered post facto and located at cosmological distances $(z \sim 0.1)$.

The discovery of iPTF16asu (Wang et al. 2017; Whitesides et al. 2017) in the intermediate Palomar Transient Factory (iPTF; Law et al. 2009) showed that at least some of these fast-luminous transients are energetic ( $\left.10^{52} \mathrm{erg}\right)$ high-velocity ("broad-lined"; $v \gtrsim 20,000 \mathrm{~km} \mathrm{~s}^{-1}$ ) stripped-envelope (Ic) supernovae (Ic-BL $\mathrm{SNe}$ ). The light curve of iPTF16asu was unusual among Ic-BL $\mathrm{SNe}$ in being inconsistent with ${ }^{56} \mathrm{Ni}$-decay (Cano 2013; Taddia et al. 2019). Suggested power sources include energy injection by a magnetar, ejecta-CSM interaction, cooling-envelope emission, and an engine-driven explosion similar to low-luminosity gamma-ray bursts - or some combination thereof. Unfortunately, the high redshift $(z=0.187)$ precluded a definitive conclusion.

Today, optical surveys such as ATLAS (Tonry et al. 2018) and the Zwicky Transient Facility (ZTF; Bellm et al. 2019a; Graham et al. 2019) have the areal coverage to discover rare transients nearby, as well as the cadence to discover transients when they are young ( $<1$ day). For example, the recent discovery of AT2018cow at $60 \mathrm{Mpc}$ (Smartt et al. 2018; Prentice et al. 2018) represented an unprecedented opportunity to study a fast-luminous optical transient up close, in detail, and in real time. Despite an intense multiwavelength observing campaign, the nature of AT2018cow remains unknownpossibilities include an engine-powered stellar explosion (Prentice et al. 2018; Ho et al. 2019; Margutti et al. 2019; Perley et al. 2019), the tidal disruption of a white dwarf by an intermediate-mass black hole (Kuin et al. 2019; Perley et al. 2019), and an electron capture SN (Lyutikov \& Toonen 2019). Regardless of the origin, it is clear that the explosion took place within dense material (Ho et al. 2019; Margutti et al. 2019; Perley et al. 2019) confined to $\lesssim 10^{16} \mathrm{~cm}$ (Ho et al. 2019).

Here we present SN2018gep, discovered as a rapidly rising $\left(1.4 \pm 0.1 \mathrm{mag} \mathrm{hr}^{-1}\right)$ and luminous $\left(M_{g \text {,peak }}=-20\right)$ transient in high-cadence data from ZTF (Ho et al. 2018c). The high inferred velocities $\left(>20,000 \mathrm{~km} \mathrm{~s}^{-1}\right)$, the spectroscopic evolution from a blue continuum to a Ic-BL SN (Costantin et al. 2018), and the rapid rise ( $t_{\text {rise }}=3$ days in $g$ band) to high peak luminosity $\left(L_{\text {bol }} \gtrsim 3 \times 10^{44} \mathrm{erg} \mathrm{s}^{-1}\right)$ all suggest that SN2018gep is a lowredshift $(z=0.03154)$ analog to iPTF16asu. The early discovery enabled an intensive follow-up campaign within the first day of the explosion, including the highest-temperature $\left(T_{\text {eff }} \gtrsim 40,000 \mathrm{~K}\right)$ spectra of a stripped-envelope $\mathrm{SN}$ to date. A retrospective search in ZTF data revealed the first definitive detection of pre-explosion activity in a Ic-BL.

The structure of the paper is as follows. We present our radio through X-ray data in Section 2. In Section 3 we outline basic properties of the explosion and its host galaxy. In Section 4 we attribute the power source for the light curve to shock breakout

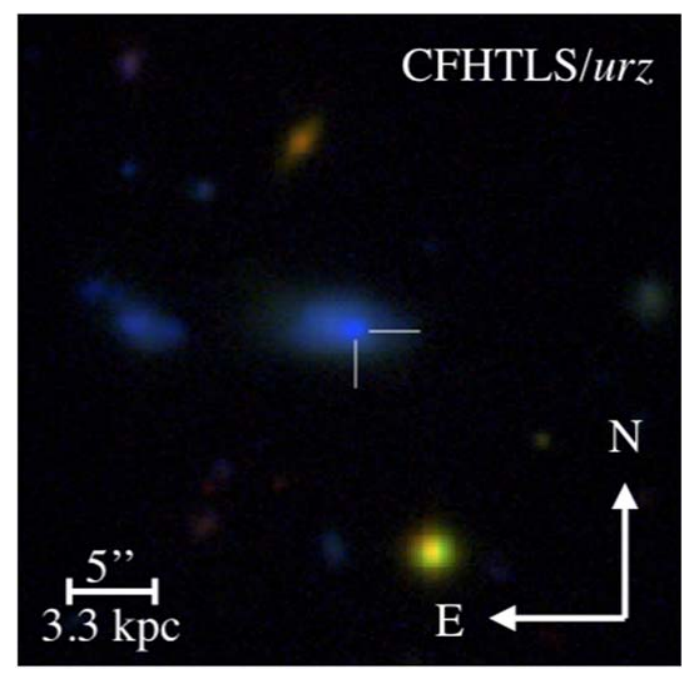

Figure 1. The position of SN2018gep (white crosshairs) in its host galaxy. Images from the Canada-France-Hawaii Telescope Legacy Survey (2004-2012), combined using the prescription in Lupton et al. (2004).

in extended CSM. In Section 5 we compare SN2018gep to unidentified fast-luminous transients at high redshift. Finally, in Section 6 we summarize our findings and look to the future. Throughout the paper, absolute times are reported in UTC and relative times are reported with respect to $t_{0}$, which is defined in Section 2.1. We assume a standard $\Lambda$ CDM cosmology (Planck Collaboration et al. 2016).

\section{Observations}

\subsection{ZTF Discovery}

ZTF observing time is divided between several different surveys, conducted using a custom mosaic camera (Dekany et al. 2016) on the 48 inch Samuel Oschin Telescope (P48) at Palomar Observatory. See Bellm et al. (2019a) for an overview of the observing system, Bellm et al. (2019b) for a description of the surveys and scheduler, and Masci et al. (2019) for details of the image processing system.

Every $5 \sigma$ point-source detection is saved as an "alert." Alerts are distributed in avro format (Patterson et al. 2019) and can be filtered based on a machine learning-based real-bogus metric (Duev et al. 2019; Mahabal et al. 2019), light-curve properties, and host characteristics (including a star-galaxy classifier; Tachibana \& Miller 2018). The ZTF collaboration uses a webbased system called the GROWTH marshal (Kasliwal et al. 2019) to identify and keep track of transients of interest.

ZTF18abukavn was discovered in an image obtained at UT 2018 September 9 03:55:18 (start of exposure) as part of the ZTF extragalactic high-cadence partnership survey, which covers $1725 \mathrm{deg}^{2}$ in six visits $(3 g, 3 r)$ per night (Bellm et al. $2019 b$ ). The discovery magnitude was $r=20.5 \pm 0.3 \mathrm{mag}$, and the source position was measured to be $\alpha=16^{\mathrm{h}} 43^{\mathrm{m}} 48.22^{\mathrm{s}}$, $\delta=+41^{\mathrm{d}} 02^{\mathrm{m}} 43.4(\mathrm{~J} 2000)$, coincident with a compact galaxy (Figure 1) at $z=0.03154$ or $d \approx 143 \mathrm{Mpc}$. As described in Section 2.3, the redshift was unknown at the time of discovery; we measured it from narrow galaxy emission lines in our follow-up spectra. The host redshift along with key observational properties of the transient are listed in Table 1.

As shown in Figure 2, the source brightened by over two magnitudes within the first three hours. These early detections passed a filter written in the GROWTH marshal that was 
Table 1

Key Observational Properties of SN2018gep and Its Host Galaxy

\begin{tabular}{|c|c|c|}
\hline Parameter & Value & Notes \\
\hline$z$ & 0.03154 & From narrow host emission lines \\
\hline$L_{\text {peak }}$ & $\gtrsim 3 \times 10^{43} \mathrm{erg}$ & $\begin{array}{r}\text { Peak UVOIR bolometric } \\
\text { luminosity }\end{array}$ \\
\hline$t_{\text {rise }}$ & $0.5-3$ days & Time from $t_{0}$ to $L_{\text {peak }}$ \\
\hline$E_{\mathrm{rad}}$ & $10^{50} \mathrm{erg}$ & UVOIR output, $\Delta t=0.5-40$ days \\
\hline$M_{r, \operatorname{prog}}$ & -15 & $\begin{array}{r}\text { Peak luminosity of pre-explosion } \\
\text { emission }\end{array}$ \\
\hline$E_{\gamma, \text { iso }}$ & $<4.9 \times 10^{48}$ & $\begin{array}{r}\text { Limit on prompt gamma-ray } \\
\text { emission from Fermi/GBM }\end{array}$ \\
\hline \multirow[t]{2}{*}{$L_{X}$} & $<2.5 \times 10^{41} \mathrm{erg} \mathrm{s}^{-1}$ & $\begin{array}{r}\text { X-ray upper limit from Swift/XRT } \\
\text { at } \Delta t=0.4-14 \text { days }\end{array}$ \\
\hline & $<10^{40} \mathrm{erg} \mathrm{s}^{-1}$ & $\begin{array}{l}\text { X-ray upper limit from Chandra at } \\
\qquad \Delta t=15 \text { and } \Delta t=70 \text { days }\end{array}$ \\
\hline$\nu L_{\nu}$ & $\approx 10^{37} \mathrm{erg} \mathrm{s}^{-1}$ & $\begin{array}{l}9 \mathrm{GHz} \text { radio luminosity from VLA } \\
\text { at } \Delta t=5 \text { and } \Delta t=16\end{array}$ \\
\hline$M_{*, \text { host }}$ & $1.3 \times 10^{8} M_{\odot}$ & Host stellar mass \\
\hline SFR $_{\text {host }}$ & $0.12 M_{\odot} \mathrm{yr}^{-1}$ & Host star formation rate \\
\hline Host metallicity & $1 / 5$ solar & Oxygen abundance on $\mathrm{O} 3 \mathrm{~N} 2$ scale \\
\hline
\end{tabular}

designed to find young SNe. We announced the discovery and fast rise via the Astronomer's Telegram (Ho et al. 2018c), and reported the object to the IAU Transient Server $\left(\mathrm{TNS}^{28}\right)$, where it received the designation SN2018gep.

We triggered ultraviolet (UV) and optical observations with the UV/Optical Telescope (UVOT; Roming et al. 2005) aboard the Neil Gehrels Swift Observatory (Gehrels et al. 2004), and observations began $10.2 \mathrm{hr}$ after the ZTF discovery (Schulze et al. 2018a). A search of IceCube data found no temporally coincident high-energy neutrinos (Blaufuss 2018).

Over the first two days, the source brightened by two additional magnitudes. A linear fit to the early $g$-band photometry gives a rise of $1.4 \pm 0.1 \mathrm{mag} \mathrm{hr}^{-1}$. This rise rate is second only to the IIb SN $16 \mathrm{gkg}$ (Bersten et al. 2018) but several orders of magnitude more luminous at discovery $\left(M_{g, \text { disc }} \approx-17 \mathrm{mag}\right)$.

To establish a reference epoch, we fit a second-order polynomial to the first three days of the $g$-band light curve in flux space, and define $t_{0}$ as the time at which the flux is zero. This gives $t_{0}$ as being $25 \pm 2$ minutes prior to the first detection, or $t_{0} \approx$ UT 2018 September 9 03:30. The physical interpretation of $t_{0}$ is not straightforward, since the light curve flattens out at early times (see Figures 2 and 3 ). We proceed using $t_{0}$ as a reference epoch but caution against assigning it physical meaning.

\subsection{Photometry}

From $\Delta t \approx 1$ day to $\Delta t \approx 60$ days, we conducted a photometric follow-up campaign at UV and optical wavelengths using Swift/UVOT, the Spectral Energy Distribution Machine (SEDM; Blagorodnova et al. 2018) mounted on the automated 60 inch telescope at Palomar (P60; Cenko et al. 2006), the optical imager (IO:O) on the Liverpool Telescope (LT; Steele et al. 2004), and the Lulin $1 \mathrm{~m}$ Telescope (LOT).

Basic reductions for the LT IO:O imaging were performed by the LT pipeline. ${ }^{29}$ Digital image subtraction and photometry for the SEDM, LT, and LOT imaging was performed using the

\footnotetext{
${ }^{28}$ https://wis-tns.weizmann.ac.il

29 https://telescope.livjm.ac.uk/TelInst/Pipelines/\#ioo
}

Fremling Automated Pipeline (FPipe; Fremling et al. 2016). Fpipe performs calibration and host subtraction against Sloan Digital Sky Survey reference images and catalogs (SDSS; Ahn et al. 2014). SEDM spectra were reduced using pysedm (Rigault et al. 2019).

The UVOT data were retrieved from the NASA Swift Data Archive $^{30}$ and reduced using standard software distributed with HEASOFT version $6.19 .^{31}$ Photometry was measured using UVOTMAGHIST with a $3^{\prime \prime}$ circular aperture. To remove the host contribution, we obtained a final epoch in all broadband filters on UT 2018 October 18 and built a host template using UVOTIMSUM and UVOTSOURCE with the same aperture used for the transient.

Figure 3 shows the full set of light curves, with a cross denoting the peak of the $r$-band light curve for reference. The position of the cross is simply the time and magnitude of our brightest $r$-band measurement, which is a good estimate given our cadence. The photometry is listed in Table 5 in Appendix A. Note that despite the steep spectral energy distribution (SED) at early times, the K-correction is minimal. We estimate that the effect is roughly $0.03 \mathrm{mag}$, which is well within our uncertainties. In Figure 4 we compare the rise time and peak absolute magnitude to other rapidly evolving transients from the literature.

\subsection{Spectroscopy}

The first spectrum was taken 0.7 day after discovery by the Spectrograph for the Rapid Acquisition of Transients (SPRAT; Piascik et al. 2014) on the LT. The spectrum showed a blue continuum with narrow galaxy emission lines, establishing this as a luminous transient $\left(M_{\mathrm{g}, \text { peak }}=-19.7\right)$. Twenty-three optical spectra were obtained from $\Delta t=0.7-61.1$ days using SPRAT, the Andalusia Faint Object Spectrograph and Camera (ALFOSC) on the Nordic Optical Telescope (NOT), the Double Spectrograph (DBSP; Oke \& Gunn 1982) on the 200 inch Hale telescope at Palomar Observatory, the Low Resolution Imaging Spectrometer (LRIS; Oke et al. 1995) on the Keck I $10 \mathrm{~m}$ telescope, and the Xinglong $2.16 \mathrm{~m}$ telescope (XLT+BFOSC) of NAOC, China (Wang et al. 2018). As discussed in Section 3.2, the early $\Delta t<5$ days spectra show broad absorption features that evolve redward with time, which we attribute to carbon and oxygen. By $\Delta t \sim 8$ days, the spectrum resembles a stripped-envelope $\mathrm{SN}$, and the usual broad features of a Ic-BL emerge (Costantin et al. 2018).

We use the automated LT pipeline reduction and extraction for the LT spectra. LRIS spectra were reduced and extracted using Lpipe (Perley 2019). The NOT spectrum was obtained at parallactic angle using a $1^{\prime \prime}$ slit, and was reduced in a standard way, including wavelength calibration against an arc lamp, and flux calibration using a spectrophotometric standard star. The XLT+BFOSC spectra were reduced using the standard IRAF routines, including corrections for bias, flat field, and removal of cosmic rays. The $\mathrm{Fe} / \mathrm{Ar}$ and $\mathrm{Fe} / \mathrm{Ne}$ arc lamp spectra obtained during the observation night are used to calibrate the wavelength of the spectra, and the standard stars observed on the same night at similar airmasses as the supernova were used to calibrate the flux of the spectra. The spectra were further corrected for continuum atmospheric extinction during flux calibration, using mean extinction curves obtained at Xinglong Observatory. Furthermore, telluric lines were removed from the data.

\footnotetext{
${ }^{30}$ https://heasarc.gsfc.nasa.gov/cgi-bin/W3Browse/swift.pl

${ }^{31}$ https://heasarc.nasa.gov/lheasoft/
} 

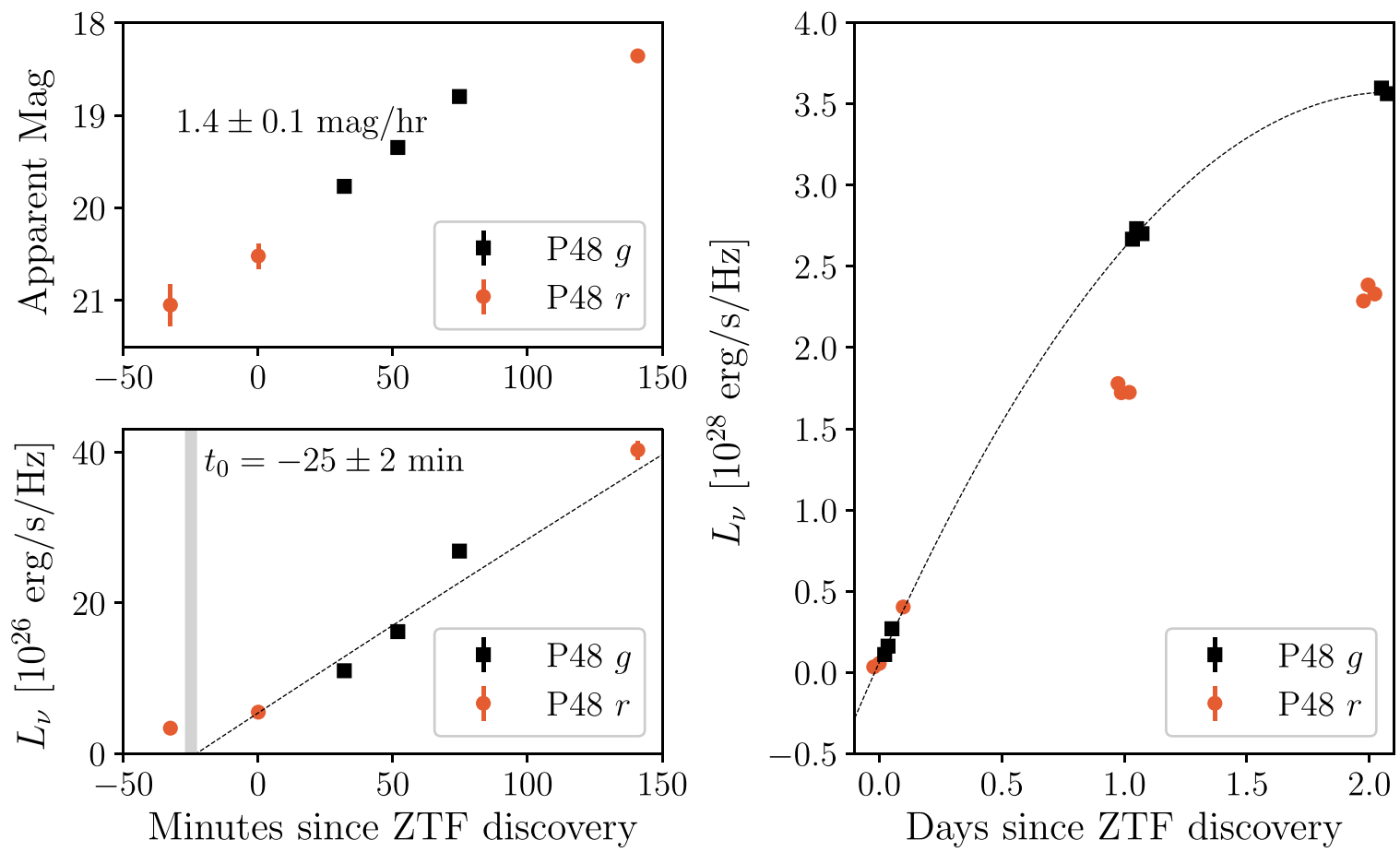

Figure 2. The rapid rise in the first few minutes and first few days after the ZTF discovery of SN2018gep. We also show an $r$-band point from prior to discovery that was found in retrospect by lowering the detection threshold from $5 \sigma$ to $3 \sigma$. Top left: the rise in magnitudes gives an almost unprecedented rate of $1.4 \pm 0.1$ mag hr ${ }^{-1}$. Bottom left: the rise in flux space together with the quadratic fit and definition of $t_{0}$. Right: the rise in flux space showing the quadratic fit.

Swift obtained three UV-grism spectra between UT 2018 September 15 3:29 and 6:58 ( $\Delta t \approx 6.4$ days) for a total exposure time of $3918 \mathrm{~s}$. The data were processed using the calibration and software described by Kuin et al. (2015). During the observation, the source spectrum was centered on the detector, which is the default location for Swift/UVOT observations. Because of this, there is second-order contamination from a nearby star, which was reduced by using a narrow extraction width (1". 3 instead of 2". 5 ). The contamination renders the spectrum unreliable at wavelengths longer than $4100 \AA$, but is negligible in the range $2850-4100 \AA$ due to absorption from the interstellar medium. Below $2200 \AA$, the spectrum overlaps with the spectrum from another star in the field of view.

The resulting spectrum (Figure 5) shows a single broad feature between $2200 \AA$ and $3000 \AA$ (rest frame). One possibility is that this is a blend of the UV features seen in superluminous supernovae (SLSNe). Line identifications for these features vary in the SLSN literature, but are typically blends of Ti III, Si III, C II, C III, and Mg II (Quimby et al. 2011; Howell et al. 2013; Mazzali et al. 2016; Yan et al. 2017).

The spectral $\log$ and a figure showing all the spectra are presented in Appendix B. In Section 3.2 we compare the early spectra to spectra at similar epochs in the literature. We model one of the early spectra, which shows a "W" feature that has been seen in SLSNe, to measure the density, density profile, and element composition of the ejecta. From the Ic-BL spectra, we measure the velocity evolution of the photosphere.

\subsection{Search for Pre-discovery Emission}

The nominal ZTF pipeline only generates detections above a $5 \sigma$ threshold. To extend the light curve further back in time, we performed forced photometry at the position of SN2018gep on single-epoch difference images from the IPAC ZTF difference imaging pipeline. The ZTF forced photometry point spread functions (PSF)-fitting code will be described in
Y. Yao et al. (2019, in preparation). As shown in Figure 2, forced photometry uncovered an earlier $3 \sigma r$-band detection.

Next, we searched for even fainter detections by constructing deeper reference images than those used by the nominal pipeline, and subtracting them from 1 to 3 day stacks of ZTF science images. The reference images were generated by performing an inverse-variance weighted coaddition of $298 R$ band and $69 \mathrm{~g}$-band images from PTF/iPTF taken between 2009 and 2016 using the CLIPPED combine strategy in SWarp (Bertin 2010; Gruen et al. 2014). PTF/iPTF images were used instead of ZTF images to build references as they were obtained years prior to the transient, and thus less likely to contain any transient flux. No cross-instrument corrections were applied to the references prior to subtraction. Pronounced regions of negative flux on the PTF/iPTF references caused by crosstalk from bright stars were masked out manually.

We stacked ZTF science images obtained between UT 2018 February 22 and 2018 August 31 in a rolling window (segregated by filter) with a width of 3 days and a period of 1 day, also using the CLIPPED technique in SWarp. Images taken between 2018 Sep 01 and $t_{0}$ were stacked in a window with a width of 1 day and a period of 1 day. Subtractions were obtained using the HOTPANTS (Becker 2015) implementation of the Alard \& Lupton (1998) PSF matching algorithm. Many of the ZTF science images during this period were obtained under exceptional conditions, and the seeing on the ZTF science coadds was often significantly better than the seeing on the PTF/iPTF references. To correct for this effect, ZTF science coadds were convolved with their own PSFs, extracted using PSFEx, prior to subtraction. During subtraction, PSF matching and convolution were performed on the template and the resulting subtractions were normalized to the photometric system of the science images. We show two example subtractions in Figure 6.

Using these newly constructed deep subtractions, PSF photometry was performed at the location of SN2018gep using the PSF 


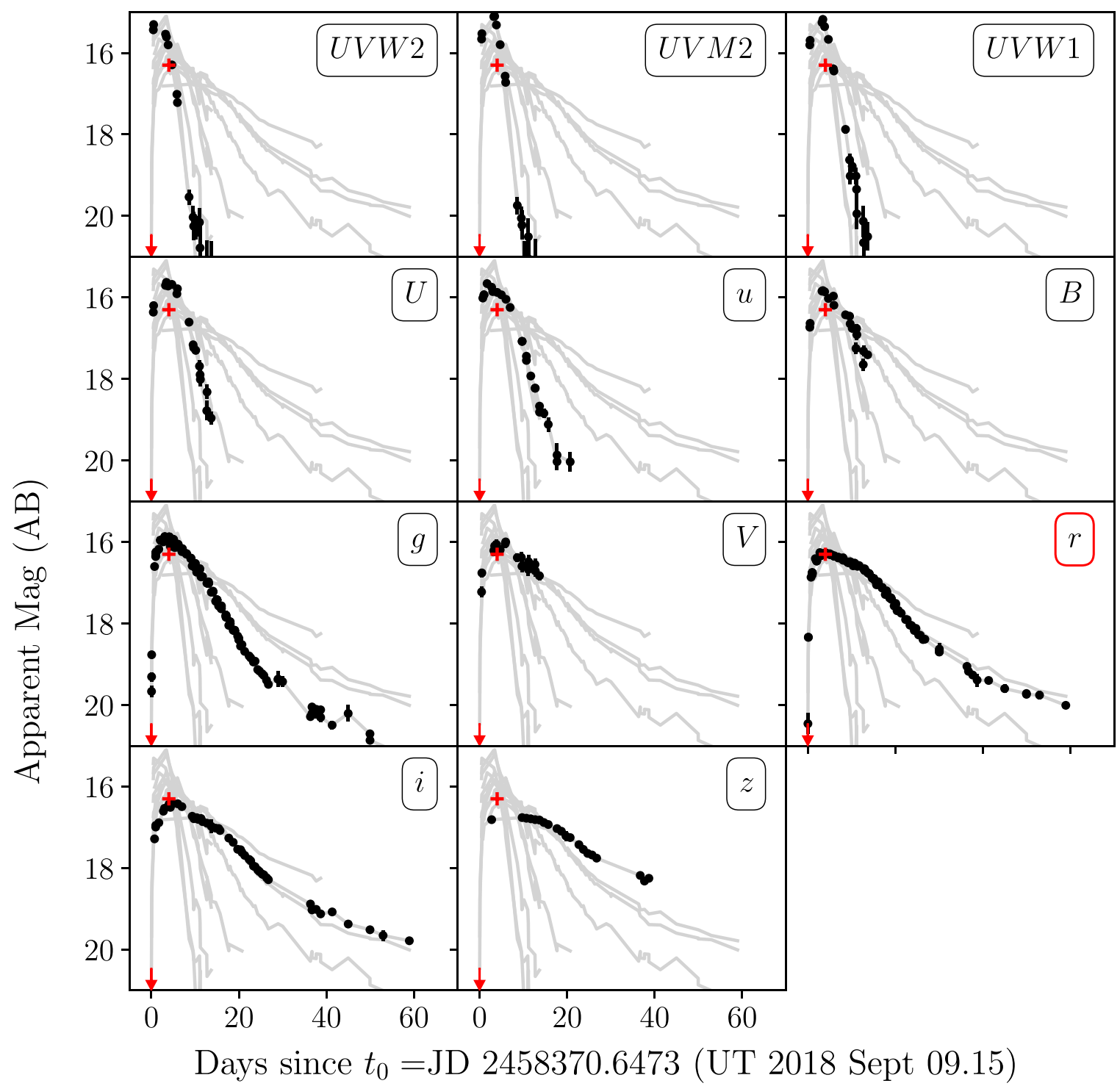

Figure 3. UV and optical light curves from Swift and ground-based facilities. The arrow marks the last nondetection, which was in $r$ band. The red cross marks the peak of the $r$-band light curve, which is 16.3 mag at $\Delta t=4$ days. The full set of light curves is shown as gray lines in the background, and each panel highlights an individual filter in black. We correct for Galactic extinction using the attenuation curve from Fitzpatrick (1999) and $E_{B-V}=R_{V} / A_{V}=0.01$ for $R_{V}=3.1$ and $A_{V}=0.029$ (Schlafly \& Finkbeiner 2011).

of the science images. To estimate the uncertainty on the flux measurements made on these subtractions, we employed a Monte Carlo technique, in which thousands of PSF fluxes were measured at random locations on the image, and the PSF-flux uncertainty was taken to be the $1 \sigma$ dispersion in these measurements. We loaded this photometry into a local instance of SkyPortal (Van der Walt et al. 2019), an open-source web application that interactively displays astronomical data sets for annotation, analysis, and discovery.

We detected significant flux excesses at the location of SN2018gep in both $g$ and $r$ bands in the weeks preceding $t_{0}$ (i.e., its first detection in single-epoch ZTF subtractions). The effective dates of these extended prediscovery detections are determined by taking an inverse-flux variance weighted average of the input image dates. The detections in the week leading up to explosion are $m_{g} \sim m_{r} \approx 22$, which is approximately the magnitude limit of the coadd subtractions. However, in an $r$-band stack of images from August 24-26 (inclusive), we detect emission at $m_{r} \sim 21.5$ at $5 \sigma$ above the background.
Assuming that the rapid rise we detected was close to the time of explosion, this is the first definitive detection of preexplosion emission in a Ic-BL SN. There was a tentative detection in another source, PTF 11qcj (Corsi et al. 2014), 1.5 and $2.5 \mathrm{yr}$ prior to the SN. In Section 4 we discuss possible mechanisms for this emission, and conclude that it is likely related to a period of eruptive mass loss immediately prior to the explosion. We note that it is unlikely that this variability arises from active galactic nucleus (AGN) activity, due to the properties of the host galaxy (Section 3.3).

With forced photometry and faint detections from stacked images and deep references, we can construct a light curve that extends weeks prior to the rapid rise in the light curve, shown in Figure 7.

\subsection{Radio Follow-up}

We observed the field of SN2018gep with the Karl G. Jansky Very Large Array (VLA) on three epochs: on 2018 September 14 UT under the Program ID VLA/18A-242 (PI: D. Perley; 


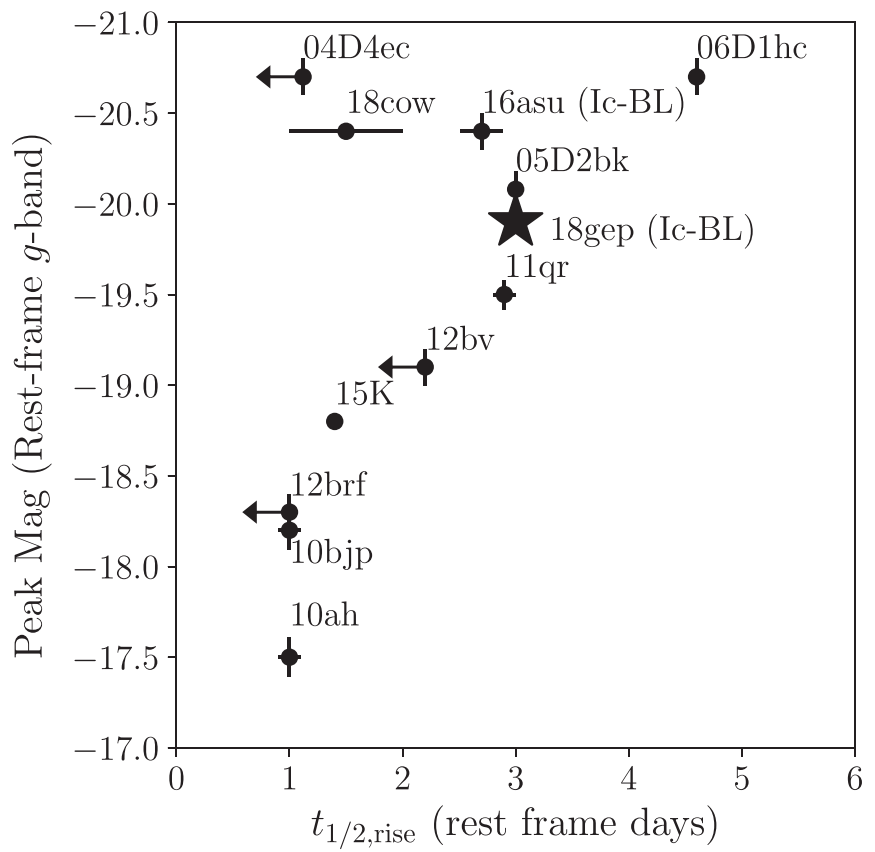

Figure 4. The rise time and peak absolute magnitude of SN2018gep, iPTF16asu (a high-redshift analog), and unclassified fast-luminous transients from Drout et al. (2014), Arcavi et al. (2016), Rest et al. (2018), and Perley et al. (2019). When possible, we report measurements in rest-frame $g$ band, and define "rise time" as time from half-max to max. For iPTF16asu, we use the quadratic fit to the early g-band light curve from Whitesides et al. (2017) as well as their reported peak magnitude, but caution that this is rest-frame $r$ band. For KSN2015K, there are only observations in the Kepler white filter (Rest et al. 2018)

Ho et al. 2018b), and on 2018 September 25 and 2018 November 23 UT under the Program ID VLA/18A-176 (PI: A. Corsi). We used 3C286 for flux calibration, and J1640+3946 for gain calibration. The observations were carried out in $\mathrm{X}$ - and $\mathrm{Ku}$-band (nominal central frequencies of $9 \mathrm{GHz}$ and $14 \mathrm{GHz}$, respectively) with a nominal bandwidth of $2 \mathrm{GHz}$. The data were calibrated using the automated VLA calibration pipeline available in the CASA package (McMullin et al. 2007) then inspected for further flagging. The CLEAN procedure (Högbom 1974) was used to form images in interactive mode. The image rms and the radio flux at the location of SN2018gep were measured using imstat in CASA. Specifically, we report the maximum flux within pixels contained in a circular region centered on the optical position of SN2018gep with radius comparable to the FWHM of the VLA synthesized beam at the appropriate frequency. The source was detected in the first two epochs, but not in the third (see Table 2). As we discuss in Section 4, the first two epochs were conducted in a different array configuration than the third epoch, and may have had a contribution from host galaxy light.

We also obtained three epochs of observations with the AMI large array (AMI-LA; Zwart et al. 2008; Hickish et al. 2018), on UT 2018 September 12, 2018 September 23, and 2018 October 20. AMI-LA is a radio interferometer comprised of eight $12.8 \mathrm{~m}$ diameter antennas that extend from $18 \mathrm{~m}$ up to $110 \mathrm{~m}$ in length and operates with a $5 \mathrm{GHz}$ bandwidth around a central frequency of $15.5 \mathrm{GHz}$.

We used a custom AMI data reduction software package reduce_dc (e.g., Perrott et al. 2013) to perform initial data reduction, flagging, and calibration of phase and flux. Phase calibration was conducted using short interleaved observations of $\mathrm{J} 1646+4059$, and for absolute flux calibration we used 3C286. Additional flagging and imaging were performed using CASA. All three observations resulted in null detections with

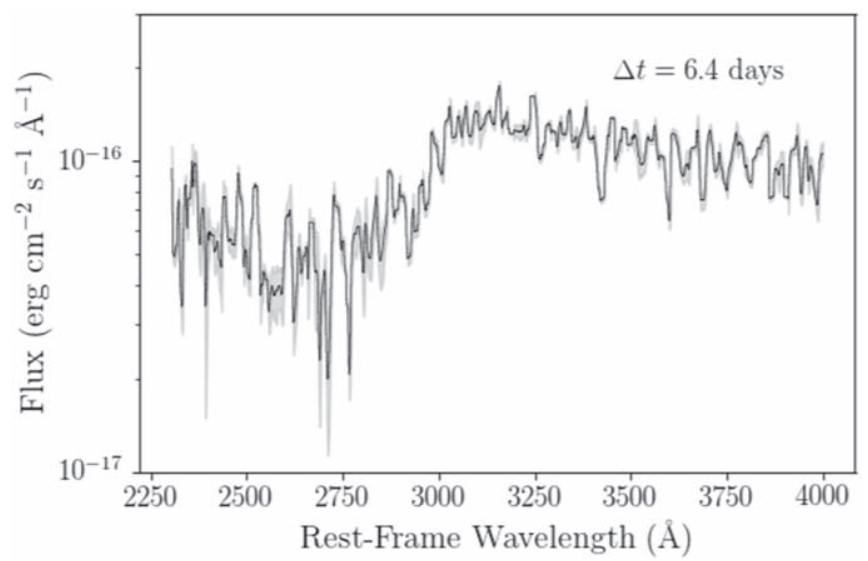

Figure 5. Swift/UVOT grism spectrum shifted to the rest frame. The black line shows the data binned such that each bin size is $10 \AA$. Light gray represents $1 \sigma$ uncertainties after binning. The spectrum has been scaled to match the UVOT $u$-band flux at this epoch (integrated from $3000 \AA$ to $3900 \AA$ ), which was determined by interpolating the Swift $u$-band light curve.

$3 \sigma$ upper limits of $\approx 120 \mu \mathrm{Jy}$ in the first two observations, and a $3 \sigma$ upper limit of $\approx 120 \mu \mathrm{Jy}$ in the last observation.

Finally, we observed at higher frequencies using the Submillimeter Array (SMA; Ho et al. 2004) on UT 2018 September 15 under its target-of-opportunity program. The project ID was 2018A-S068. Observations were performed in the sub-compact configuration using seven antennas. The observations were performed using RxA and $\mathrm{RxB}$ receivers tuned to $\mathrm{LO}$ frequencies of $225.55 \mathrm{GHz}$ and $233.55 \mathrm{GHz}$, respectively, providing $32 \mathrm{GHz}$ of continuous bandwidth ranging from 213.55 to $245.55 \mathrm{GHz}$ with a spectral resolution of $140.0 \mathrm{kHz}$ per channel. The atmospheric opacity was around $0.16-0.19$ with system temperatures around $100-200 \mathrm{~K}$. The nearby quasars $1635+381$ and $3 \mathrm{C} 345$ were used as the primary phase and amplitude gain calibrators with absolute flux calibration performed by comparison to Neptune. Passband calibration was derived using 3C454.3. Data calibration was performed using the MIR IDL package for the SMA, with subsequent analysis performed in MIRIAD (Sault et al. 1995). For the flux measurements, all spectral channels were averaged together into a single continuum channel and an rms of $0.6 \mathrm{mJy}$ was achieved after 75 minutes on-source.

The full set of radio and submillimeter measurements are listed in Table 2.

\subsection{X-Ray Follow-up}

We observed the position of SN2018gep with Swift/XRT from $\Delta t \approx 0.4-14$ days. The source was not detected in any epoch. To measure upper limits, we used web-based tools developed by the Swift-XRT team (Evans et al. 2009). For the first epoch, the $3 \sigma$ upper limit was $0.003 \mathrm{ct} \mathrm{s}^{-1}$. To convert the upper limit from count rate to flux, we assumed ${ }^{32}$ a Galactic neutral hydrogen column density of $1.3 \times 10^{20} \mathrm{~cm}^{-2}$, and a power-law spectrum with photon index $\Gamma=2$. This gives ${ }^{33}$ an unabsorbed $0.3-10 \mathrm{keV}$ flux of $<9.9 \times 10^{-14} \mathrm{erg} \mathrm{cm}^{-2} \mathrm{~s}^{-1}$, and $L_{X}<2.5 \times 10^{41} \mathrm{erg} \mathrm{s}^{-1}$.

We obtained two epochs of observations with the Advanced CCD Imaging Spectrometer (Garmire et al. 2003) on the Chandra $X$-ray Observatory via our approved program (Proposal No.

\footnotetext{
32 https://heasarc.gsfc.nasa.gov/cgi-bin/Tools/w3nh/w3nh.pl

33 https://heasarc.gsfc.nasa.gov/cgi-bin/Tools/w3pimms/w3pimms.pl
} 
r-band, 2018-08-24-2018-08-26
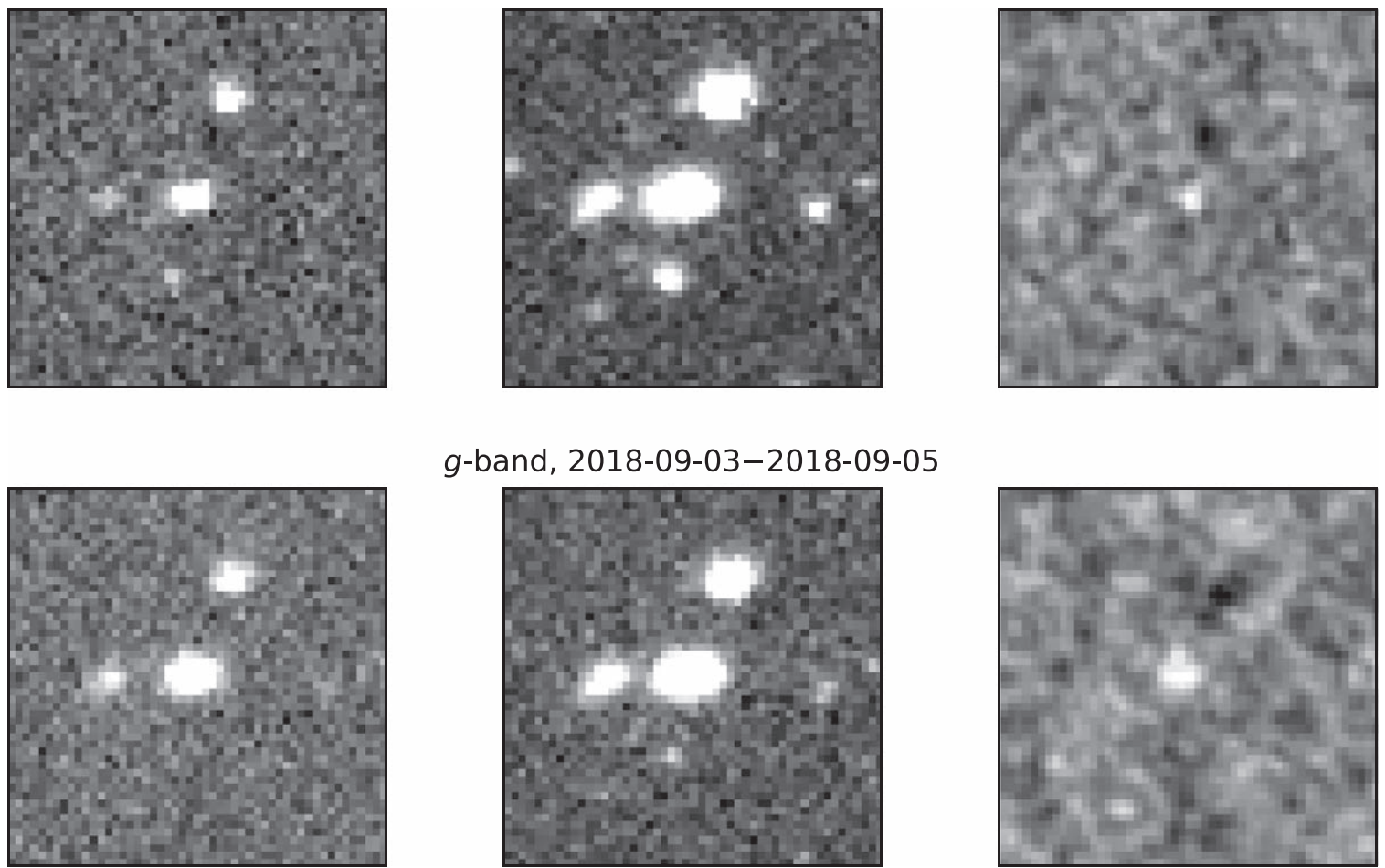

Figure 6. Sample pre-explosion subtractions of deep PTF/iPTF references from ZTF science images stacked in 3 days bins (see Section 2.4). Each cutout is centered on the location of SN2018gep. The subtractions show clear emission at the location of the SN in both $g$ and $r$ bands days to weeks before the discovery of the SN in ZTF.

19500451; PI: Corsi). The first epoch began at 9:25 UTC on 2018 10 October $(\Delta t \approx 15$ days) under ObsId 20319 (integration time $12.2 \mathrm{ks}$ ), and the second began at 21:31 UTC on 2018 December 4 ( $\Delta t \approx 70$ days) under ObsId 20320 (integration time $12.1 \mathrm{ks}$ ). No $\mathrm{X}$-ray emission is detected at the location of SN2018gep in either epoch, with $90 \%$ upper limits on the $0.5-7.0 \mathrm{keV}$ count rate of $\approx 2.7 \times 10^{-4} \mathrm{ct} \mathrm{s}^{-1}$. Using the same values of hydrogen column density and power-law photon index as in our XRT measurements, we find upper limits on the unabsorbed $0.5-7 \mathrm{keV} \mathrm{X}$-ray flux of $<3.2 \times 10^{-15} \mathrm{erg} \mathrm{cm}^{-2} \mathrm{~s}^{-1}$, or (for a direct comparison to the XRT band) a $0.3-10 \mathrm{keV}$ X-ray flux of $<4.2 \times 10^{-15}$

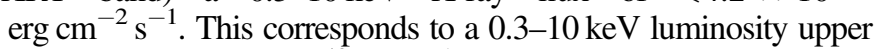
limit of $L_{X}<1.0 \times 10^{40} \mathrm{erg} \mathrm{s}^{-1}$.

\subsection{Search for Prompt Gamma-Ray Emission}

We created a tool to search for prompt gamma-ray emission (GRBs) from Fermi-Gamma-ray Burst Monitor (GBM) (Gruber et al. 2014; von Kienlin et al. 2014; Narayana Bhat et al. 2016), the Swift Burst Alert Telescope (BAT; Barthelmy et al. 2005), and the IPN, which we have made available online. ${ }^{34}$ We did not find any GRB consistent with the position and $t_{0}$ of SN2018gep.

Our tool also determines whether a given position was visible to BAT and GBM at a given time, using the spacecraft pointing history. We use existing $\operatorname{code}^{35}$ to determine the BAT history. We find that the position of SN2018gep was in the BAT field of view from UTC 03:13:40 to 03:30:38, before Swift slewed to another location.

We also find that at $t_{0}$ SN2018gep was visible to the Fermi GBM (Meegan et al. 2009). We ran a targeted GRB search in $10-1000 \mathrm{keV} \mathrm{Fermi/GBM}$ data from three hours prior to $t_{0}$ to

\footnotetext{
34 https://github.com/annayqho/HE_Burst_Search

35 https://github.com/lanl/swiftbat_python
}

half an hour after $t_{0}$. We use the soft template, which is a smoothly broken power law with low-energy index -1.9 and high-energy index -2.7 , and an SED peak at $70 \mathrm{keV}$. The search methodology (and parameters of the other templates) are described in Blackburn et al. (2015) and Goldstein et al. (2016). No signals with a consistent location were found. For the $100 \mathrm{~s}$ integration time, the fluence upper limit is $2 \times 10^{-6} \mathrm{erg} \mathrm{cm}^{-2}$. This limit corresponds to a $10-1000 \mathrm{keV}$ isotropic energy release of $E_{\gamma \text {,iso }}<4.9 \times 10^{48} \mathrm{erg}$. Limits for different spectral templates and integration times are shown in Figure 8.

\subsection{Host Galaxy Data}

We measure line fluxes using the Keck optical spectrum obtained at $\Delta t \approx 61$ days (Figure 25). We model the local continuum with a low-order polynomial and each emission line by a Gaussian profile of FHWM $\sim 5.3 \AA$. This is appropriate if Balmer absorption is negligible, which is generally the case for starburst galaxies. For the host of SN2018gep, the Balmer decrement between $\mathrm{H} \beta, \mathrm{H} \gamma$, and $\mathrm{H} \delta$ does not show any excess with respect to the expected values in Osterbrock \& Ferland (2006). The resulting line fluxes are listed in Table 7.

We retrieved archival images of the host galaxy from Galaxy Evolution Explorer (GALEX) Data Release (DR) 8/9 (Martin et al. 2005), SDSS DR9 (Ahn et al. 2012), Panoramic Survey Telescope And Rapid Response System (PanSTARRS, PS1) DR1 (Chambers et al. 2016), Two-Micron All Sky Survey (2MASS; Skrutskie et al. 2006), and Wide-Field Infrared Survey Explorer (WISE; Wright et al. 2010). We also used UVOT photometry from Swift, and NIR photometry from the Canada-France-Hawaii Telescope Legacy Survey (CFHTLS; Hudelot et al. 2012).

The images are characterized by different pixel scales (e.g., SDSS 0. 40/px, GALEX $1^{\prime \prime} / \mathrm{px}$ ) and different point spread 

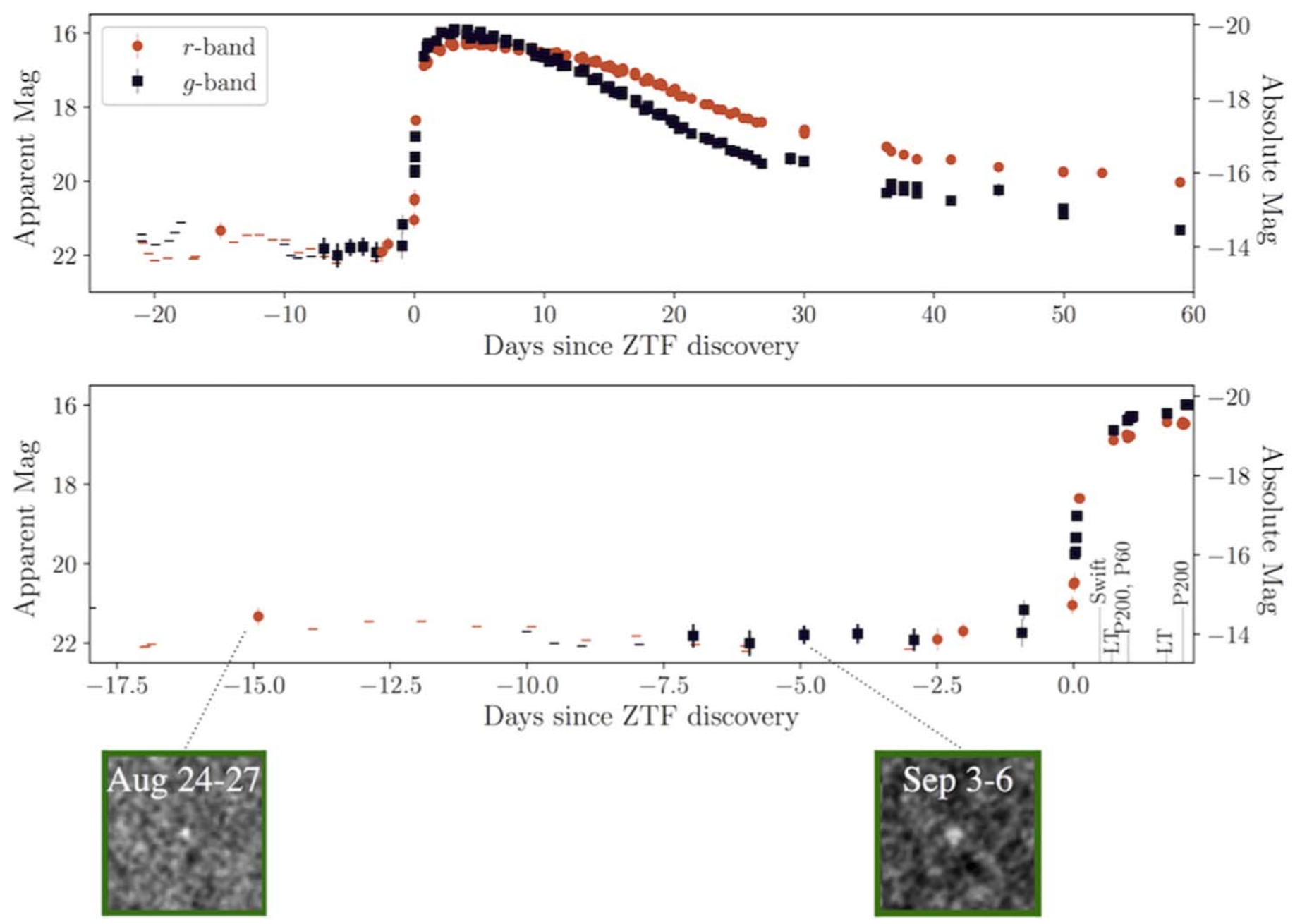

Figure 7. Full $r$ and $g$-band light curves of SN2018gep. Horizontal lines show $3 \sigma$ upper limits. Points at $t<0$ are from 3 days stacks of ZTF/P48 data as described in Section 2.4. Sample subtractions from two of these stacks are shown in the bottom row.

functions (e.g., SDSS/PS1 1"-2", WISE/W2 6!"5). To obtain accurate photometry, we use the matched-aperture photometry software package LAMBDA ADAPTIVE MULTI-BAND DEBLENDING ALGORITHM IN R (LAMBDAR; Wright et al. 2016) that is based on a photometry software package developed by Bourne et al. (2012). To measure the total flux of the host galaxy, we defined an elliptical aperture that encircles the entire galaxy in the SDSS $/ r^{\prime}$-band image. This aperture was then convolved in LAMBDAR with the PSF of a given image that we specified directly (GALEX and WISE data) or that we approximated by a two-dimensional Gaussian (2MASS, SDSS and PS1 images). After instrumental magnitudes were measured, we calibrated the photometry against instrumentspecific zero-points (GALEX, SDSS, and PS1 data), or, as in the case of 2MASS and WISE images, against a local sequence of stars from the 2MASS Point Source Catalogue and the AllWISE catalog. The photometry from the UVOT images was extracted with the command UVOTSOURCE in HEASOFT and a circular aperture with a radius of $8^{\prime \prime}$. The photometry of the CFHT/WIRCAM data was performed with the software tool presented in Schulze et al. (2018b). ${ }^{36}$ To convert the 2MASS, UVOT, WIRCAM, and WISE photometry to the AB system, we applied the offsets reported in Blanton \& Roweis (2007),

$\overline{36}$ https://github.com/steveschulze/aperture_photometry
Breeveld et al. (2011), and Cutri et al. (2013). The resulting photometry is summarized in Table 8 .

\section{Basic Properties of the Explosion and Its Host Galaxy}

The observations we presented in Section 2 constitute some of the most detailed early time observations of a strippedenvelope SN to date. In this section we use this data to derive basic properties of the explosion: the evolution of bolometric luminosity, radius, and effective temperature over time (Section 3.1), the velocity evolution of the photosphere and the density and composition of the ejecta as measured from the spectra (Section 3.2), and the mass, metallicity, and star formation rate (SFR) of the host galaxy (Section 3.3). These properties are summarized in Table 1.

\subsection{Physical Evolution from Blackbody Fits}

By interpolating the UVOT and ground-based photometry, we construct multiband SEDs and fit a Planck function on each epoch to measure the evolution of luminosity, radius, and effective temperature. To estimate the uncertainties, we perform a Monte Carlo simulation with 600 trials, each time adding noise corresponding to a $15 \%$ systematic uncertainty on each data point, motivated by the need to obtain a combined $\chi^{2} /$ dof $\sim 1$ across all epochs. The uncertainties for each parameter are taken 
Table 2

Radio Flux Density Measurements for SN2018gep

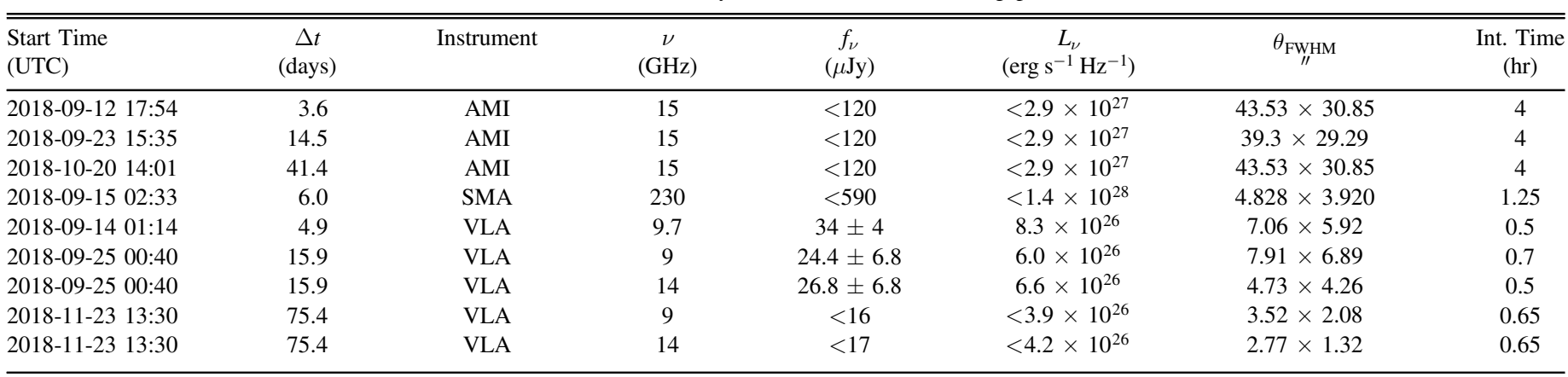

Note. For VLA measurements: the quoted errors are calculated as the quadrature sums of the image rms, plus a 5\% nominal absolute flux calibration uncertainty. When the peak flux density within the circular region is less than three times the rms, we report an upper limit equal to three times the rms of the image. For AMI measurements: nondetections are reported as $3 \sigma$ upper limits. For SMA measurements: nondetections are reported as a $1 \sigma$ upper limit.

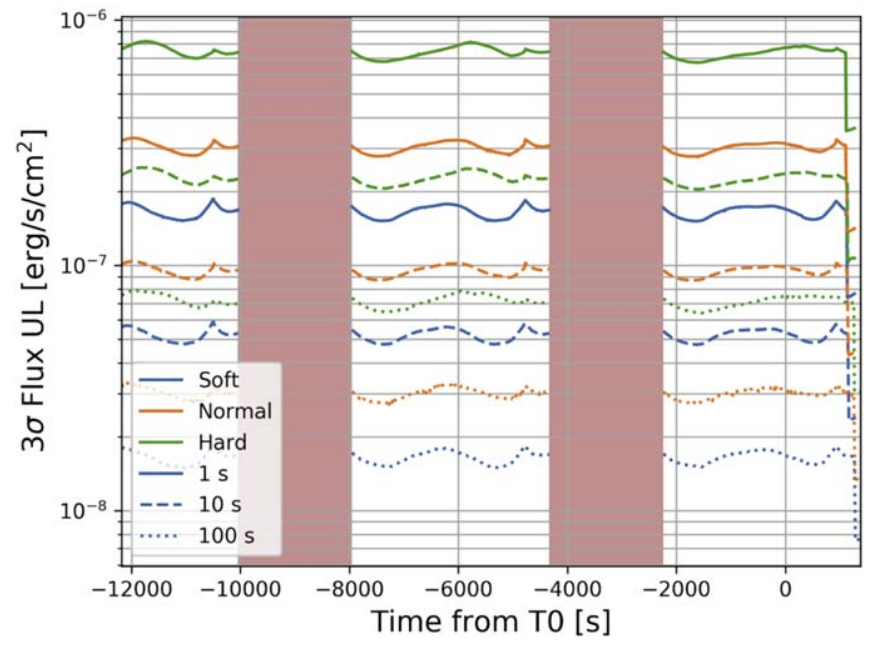

Figure 8. $3 \sigma$ upper limits from GBM GRB search, which we performed for three hours prior to $t_{0}$. The red vertical bars indicate epochs when GBM was not taking data due to passing through the South Atlantic Anomaly. The time of $t_{0}$ was estimated from a fit to the early data (Figure 7), and is $26 \pm 5$ minutes prior to the first detection.

as the 16-to- 84 percentile range from this simulation. The SED fits are shown in Appendix A, and the resulting evolution in bolometric luminosity, photospheric radius, and effective temperature is listed in Table 3. We plot the physical evolution in Figure 9, with a comparison to iPTF16asu and AT2018cow.

The bolometric luminosity peaks between $\Delta t=0.5$ day and $\Delta t=3$ days, at $>3 \times 10^{44} \mathrm{erg} \mathrm{s}^{-1}$. In Figure 10 we compare this peak luminosity and time to peak luminosity with several classes of stellar explosions. As in iPTF16asu, the bolometric luminosity falls as an exponential at late times $(t>10$ days). The total integrated UV and optical $(\approx 2000-9000 \AA)$ blackbody energy output from $\Delta t=0.5-40$ days is $\sim 10^{50} \mathrm{erg}$, similar to that of iPTF16asu. The earliest photospheric radius we measure is $\sim 20$ au, at $\Delta t=0.05$ day. Until $\Delta t \approx 17$ days the radius expands over time with a very large inferred velocity of $v \approx 0.1 c$. After that, it remains flat, and even appears to recede. This possible recession corresponds to a flattening in the temperature at $\sim 5000 \mathrm{~K}$, which is the recombination temperature of carbon and oxygen. This effect was not seen in iPTF16asu, which remained hotter (and more luminous) for longer. Finally, the effective temperature rises before falling as $\sim t^{-1}$. We interpret these properties in the context of shock-cooling emission in Section 4.
Table 3

Physical Evolution of AT2018gep from Blackbody Fits

\begin{tabular}{lccc}
\hline \hline$\Delta t$ & $L\left(10^{10} L_{\odot}\right)$ & $R(\mathrm{au})$ & $T(k \mathrm{~K})$ \\
\hline 0.05 & $0.04_{-0.02}^{+0.04}$ & $21_{-6}^{+14}$ & $13_{-4}^{+5}$ \\
0.48 & $7.4_{-4.1}^{+8.6}$ & $22_{-5}^{+7}$ & $46_{-13}^{+16}$ \\
0.73 & $4.5_{-2.8}^{+5.8}$ & $31_{-6}^{+11}$ & $35_{-11}^{+12}$ \\
1.0 & $2.2_{-1.2}^{+2.1}$ & $46_{-9}^{+18}$ & $24_{-6}^{+6}$ \\
1.7 & $3.5_{-2.1}^{+4.2}$ & $46_{-10}^{+22}$ & $27_{-8}^{+9}$ \\
2.7 & $1.3_{-0.4}^{+1.2}$ & $78_{-20}^{+22}$ & $16_{-3}^{+5}$ \\
3.2 & $3.5_{-1.3}^{+2.2}$ & $50_{-8}^{+14}$ & $26_{-5}^{+6}$ \\
3.8 & $2.9_{-0.8}^{+1.7}$ & $56_{-11}^{+11}$ & $23_{-3}^{+5}$ \\
4.7 & $1.7_{-0.7}^{+0.7}$ & $69_{-14}^{+16}$ & $18_{-2}^{+3}$ \\
5.9 & $0.88_{-0.08}^{+0.17}$ & $100_{-21}^{+14}$ & $13_{-0}^{+1}$ \\
8.6 & $0.46_{-0.06}^{+0.08}$ & $220_{-39}^{+46}$ & $7.4_{-0.5}^{+0.6}$ \\
9.6 & $0.33_{-0.03}^{+0.04}$ & $200_{-24}^{+33}$ & $7.1_{-0.4}^{+0.4}$ \\
10.0 & $0.31_{-0.03}^{+0.04}$ & $210_{-28}^{+34}$ & $6.9_{-0.4}^{+0.4}$ \\
11.0 & $0.28_{-0.03}^{+0.04}$ & $220_{-35}^{+33}$ & $6.5_{-0.3}^{+0.3}$ \\
13.0 & $0.25_{-0.03}^{+0.03}$ & $260_{-42}^{+50}$ & $5.8_{-0.3}^{+0.3}$ \\
14.0 & $0.22_{-0.03}^{+0.04}$ & $270_{-47}^{+60}$ & $5.5_{-0.3}^{+0.4}$ \\
16.0 & $0.17_{-0.04}^{+0.04}$ & $260_{-58}^{+76}$ & $5.3_{-0.5}^{+0.5}$ \\
18.0 & $0.15_{-0.02}^{+0.04}$ & $300_{-64}^{+77}$ & $4.7_{-0.4}^{+0.4}$ \\
21.0 & $0.11_{-0.02}^{+0.03}$ & $250_{-58}^{+83}$ & $4.7_{-0.4}^{+0.4}$ \\
25.0 & $0.073_{-0.013}^{+0.02}$ & $240_{-85}^{+95}$ & $4.5_{-0.5}^{+0.9}$ \\
38.0 & $0.034_{-0.007}^{+0.012}$ & $180_{-55}^{+86}$ & $4.2_{-0.5}^{+0.6}$ \\
\hline
\end{tabular}

(This table is available in machine-readable form.)

\subsection{Spectral Evolution and Velocity Measurements}

\subsubsection{Comparisons to Early Spectra in the Literature}

We obtained nine spectra of SN2018gep in the first five days after discovery. These early spectra are shown in Figure 11, when the effective temperature declined from $50,000 \mathrm{~K}$ to $20,000 \mathrm{~K}$. To our knowledge, our early spectra have no analogs in the literature, in that there has never been a spectrum of a strippedenvelope $\mathrm{SN}$ at such a high temperature (excluding spectra during the afterglow phase of GRBs). ${ }^{37}$ Two of the earliest spectra in the literature, one at $\Delta t=2$ days for Type Ic SN PTF10vgv (Corsi et al. 2012) and one at $\Delta t=3$ days for Type Ic SN PTF12gzk (Ben-Ami et al. 2012) are redder and exhibit more features than

\footnotetext{
37 There is however a spectrum of a Type II SN at a comparable temperature: iPTF13dqy was $\sim 50,000 \mathrm{~K}$ at the time of the first spectrum (Yaron et al. 2017).
} 

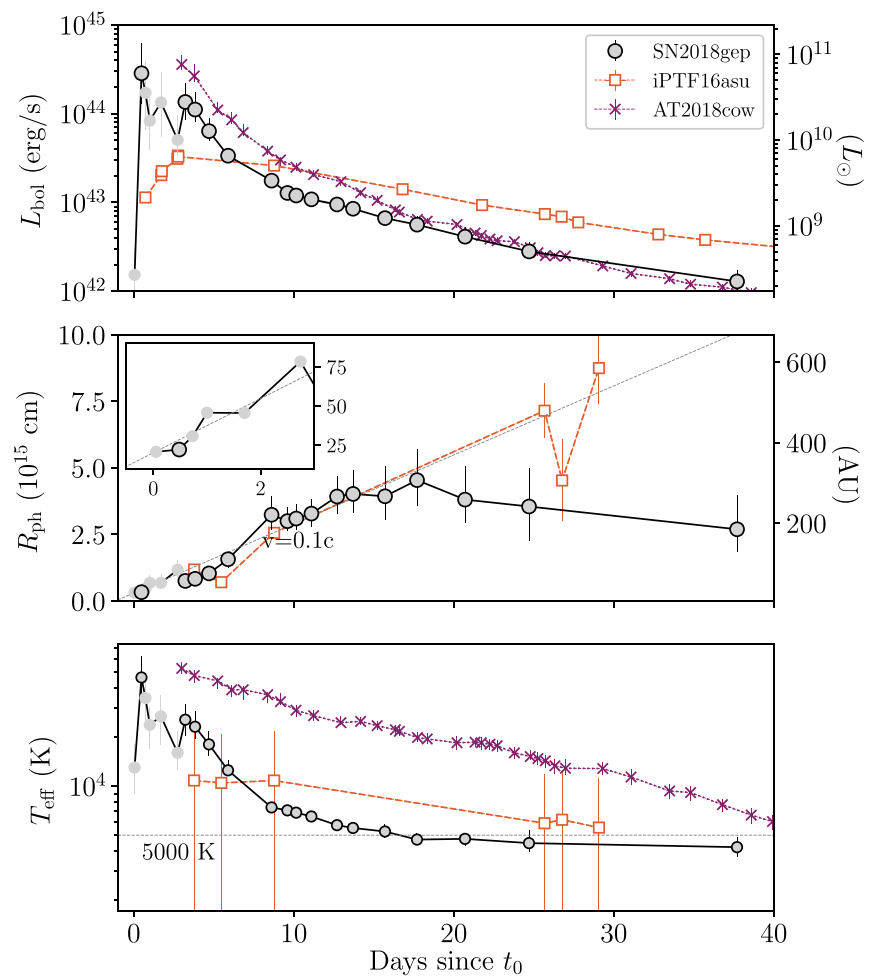

Figure 9. Evolution of blackbody properties (luminosity, radius, temperature) over time compared to the Ic-BL SN iPTF16asu and the fast-luminous optical transient AT2018cow. The light gray circles are derived from optical data only. The outlined circles are derived from UV and optical data. Middle panel: dotted line shows $v=0.1 c$. Note that $R \neq 0$ at $t_{0}$, and instead $R(t=0)=3 \times 10^{14} \mathrm{~cm}$. Due to the scaling of our plot we do not show the radius evolution of AT2018cow, which drops from $8 \times 10^{14} \mathrm{~cm}$ to $10^{14} \mathrm{~cm}$ on this timescale. Bottom panel: dotted horizontal line shows $5000 \mathrm{~K}$, the recombination temperature for carbon and oxygen. Once this temperature is reached, the photosphere flattens out (and potentially begins to recede).

the spectrum of SN2018gep. We show the comparison in Figure 11.

At $\Delta t \approx 4$ days, a "W" feature emerges in the rest-frame wavelength range $3800-4350 \AA$. In the second-from-bottom panel of Figure 11 we make a comparison to "W" features seen in SN 2008D (e.g., Modjaz et al. 2009), which was a Type Ib SN associated with an X-ray flash (Mazzali et al. 2008), and in a typical pre-max stripped-envelope SLSN (Type I SLSN; Moriya et al. 2018; Gal-Yam 2019b). The absorption lines are broadened much more than in PTF12dam (Nicholl et al. 2013) and probably more than in SN2008D as well. Finally, SN2018gep cooled more slowly than SN2008D: only after 4.25 days did it reach the temperature that SN 2008D reached after $>2$ days.

\subsubsection{Origin of the " $W$ " feature}

The lack of comparison data at such early epochs (high temperatures) motivated us to model one of the early spectra in order to determine the composition and density profile of the ejecta. We used the spectral synthesis code JEKYLL (Ergon et al. 2018), configured to run in steady state using a full NLTE solution. An inner blackbody boundary was placed at a high continuum optical depth $(\sim 50)$, and the temperature at this boundary was iteratively determined to reproduce the observed luminosity. The atomic data used is based on what was specified in Ergon et al. (2018), but has been extended as described in Appendix C. We explored models with $\mathrm{C} / \mathrm{O}$ (mass fractions: $0.23 / 0.65$ ) and $\mathrm{O} / \mathrm{Ne} / \mathrm{Mg}$ (mass fractions: $0.68 / 0.22 / 0.07$ )

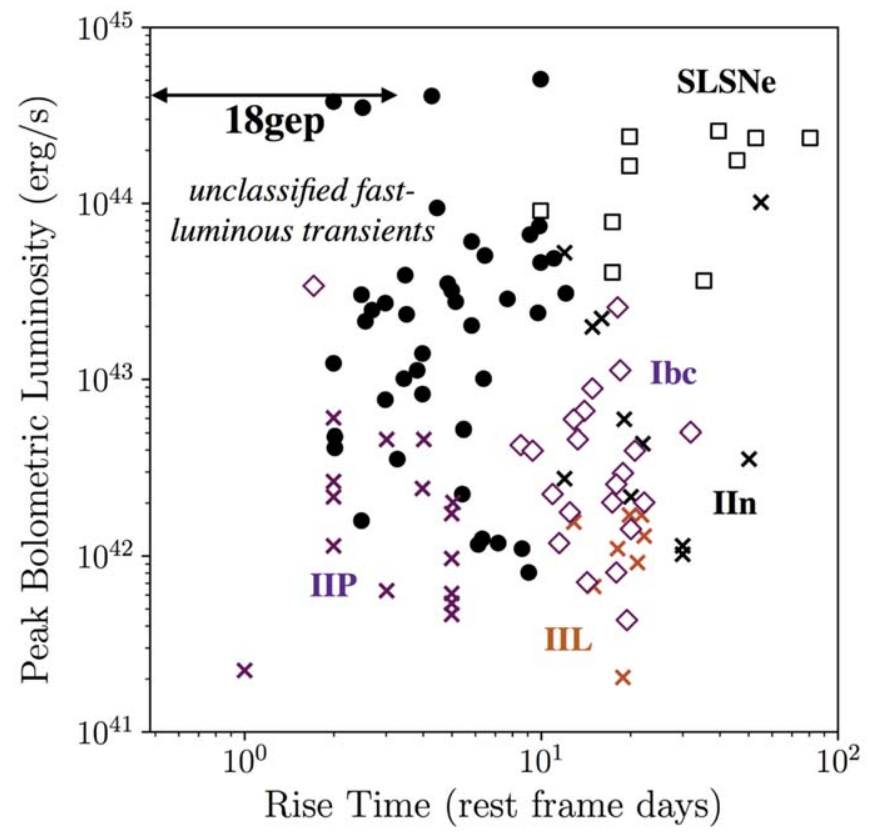

Figure 10. Rise to peak bolometric luminosity compared to other classes of transients. Modified from Figure 1 in Margutti et al. (2019).

compositions taken from a model by Woosley \& Heger $(2007)^{38}$ and a power-law density profile, where the density at the inner border was adjusted to fit the observed line velocities. Except for the density at the inner border, various power-law indices were also explored, but in the end an index of -9 worked out best.

Figures 12 and 13 show the model with the best overall agreement with the spectra and the SED (as listed in Table 6 the spectrum was obtained at high airmass, making it difficult to correct for telluric features). The model has a $\mathrm{C} / \mathrm{O}$ composition, an inner border at $22,000 \mathrm{~km} \mathrm{~s}^{-1}$ (corresponding to an optical depth of $~ 50$ ), a density of $4 \times 10^{-12} \mathrm{~g} \mathrm{~cm}^{-3}$ at this border, and a density profile with a power-law index of -9 . In Figure 12 we show that the model does a good job of reproducing both the spectrum and the SED of SN2018gep. In particular, it is interesting to note that the "W" feature seems to arise naturally in $\mathrm{C} / \mathrm{O}$ material at the observed conditions. A similar conclusion was reached by Dessart (2019), whose magnetar-powered SLSN-I models, calculated using the NLTE code CMFGEN, show the "W" feature even when nonthermal processes were not included in the calculation (as in our case).

In the model, the "W" feature mainly arises from the O II $2 \mathrm{p}^{2}(3 \mathrm{P}) 3 \mathrm{~s} 4 \mathrm{P} \leftrightarrow 2 \mathrm{p}^{2}(3 \mathrm{P}) 3 \mathrm{p} 4 \mathrm{D}^{\circ}(4639-4676 \AA)$, O II $2 \mathrm{p}^{2}(3 \mathrm{P}) 3 \mathrm{~s}$ $4 \mathrm{P} \leftrightarrow 2 \mathrm{p}^{2}(3 \mathrm{P}) 3 \mathrm{p} 2 \mathrm{D} \quad(4649 \AA)$, and O II $2 \mathrm{p}^{2}(3 \mathrm{P}) 3 \mathrm{~s} \quad 4 \mathrm{P} \leftrightarrow$ $2 \mathrm{p}^{2}(3 \mathrm{P}) 3 \mathrm{p} 4 \mathrm{P}^{\circ}(4317-4357 \AA)$ transitions. The departure from LTE is modest in the line-forming region, and the departure coefficients for the $\mathrm{O}$ II states are small. The spectrum redward of the "W" feature is shaped by carbon lines, and the features near 5700 and $6500 \AA$ arise from the $\mathrm{C}$ II $3 \mathrm{~s} 2 \mathrm{~S} \leftrightarrow 3 \mathrm{p} 2 \mathrm{P}^{\circ}$ (6578, $6583 \AA$ ) and C III 2s3p 1 $\mathrm{P}^{\circ} \leftrightarrow 2 \mathrm{~s} 3 \mathrm{~d} 1 \mathrm{D}(5696 \AA)$ transitions, respectively. In the model, the $\mathrm{C}$ II feature is too weak, suggesting that the ionization level is too high in the model. There is also a contribution from the C III $2 \mathrm{~s} 3 \mathrm{~s} 3 \mathrm{~S} \leftrightarrow 2 \mathrm{~s} 3 \mathrm{p} 3 \mathrm{P}^{\circ}$ (4647-4651 $\AA$ ) transition to the red part of the "W" feature, which could potentially be what is seen in the spectra from earlier epochs. In

\footnotetext{
38 The model was divided into compositional zones by Jerkstrand et al. (2015) and a detailed specification of the $\mathrm{C} / \mathrm{O}$ and $\mathrm{O} / \mathrm{Ne} / \mathrm{Mg}$ zones is given in Table D. 2 therein.
} 

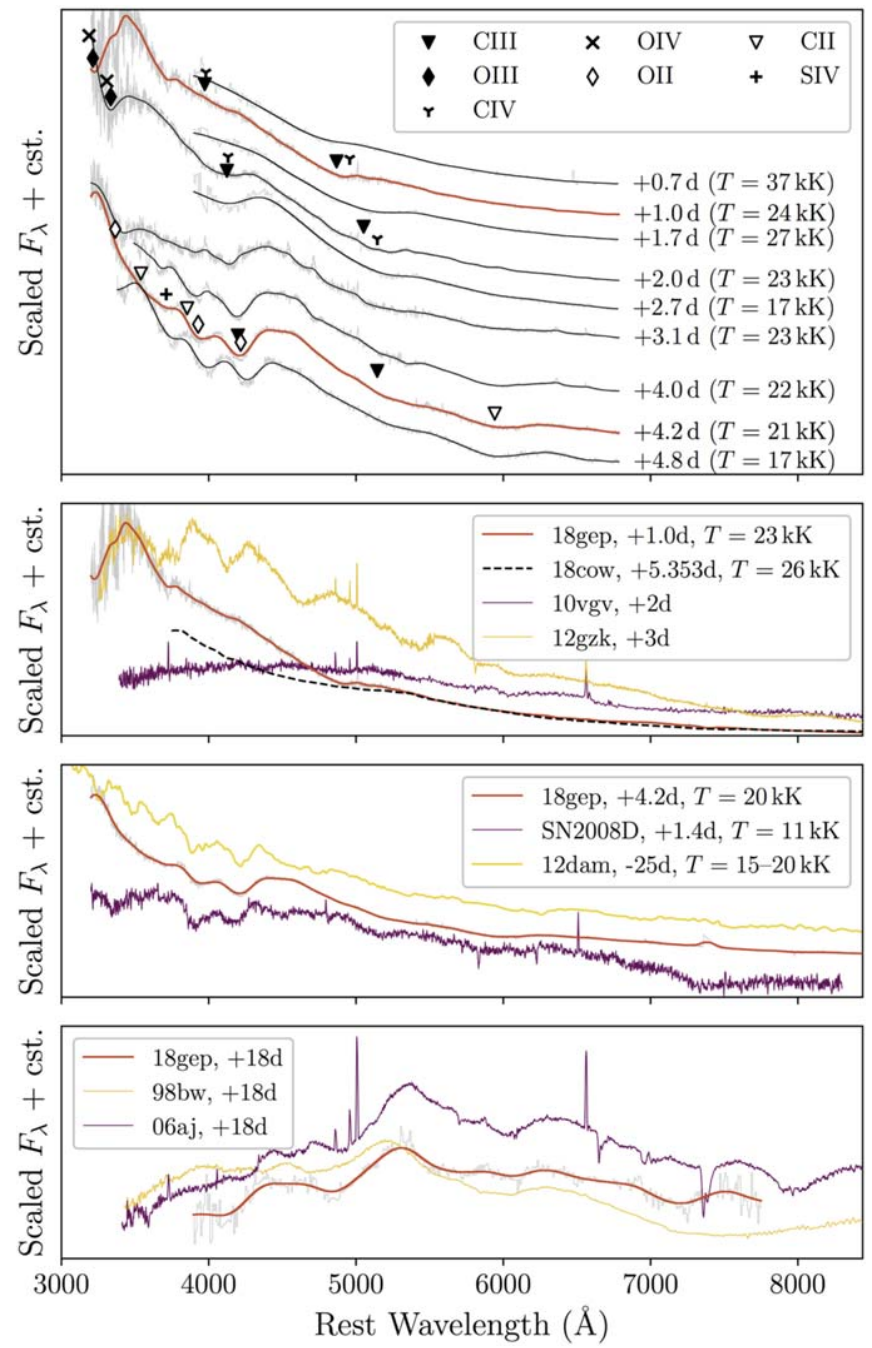

Figure 11. Top panel: spectra of SN2018gep taken in the first five days. Broad absorption features are consistent with ionized carbon and oxygen, which evolve redward with time. Second-from-top panel: an early spectrum of $18 \mathrm{gep}$ compared to spectra from other stellar explosions at a comparable phase. Second-from-bottom panel: the spectrum at $\Delta t=4.2$ days shows a "W" feature, which we compare to similar "W" features seen in an early spectrum of SN2008D from Modjaz et al. (2009), and a typical pre-max spectrum of a SLSN-I (PTF12dam, from Nicholl et al. 2013). We boost the SLSN spectrum by an additional expansion velocity of $\sim 15,000 \mathrm{~km} \mathrm{~s}^{-1}$, and apply reddening of $E(B-V)=0.63$ to SN 2008D. Weak features in the red are also similar to what are seen in PTF12dam, and are consistent with arising from CII and CIII lines, following the analysis of Gal-Yam (2019a). The lack of narrow carbon features as well as the smooth spectrum below $3700 \AA$ A suggest a large velocity dispersion leading to significant line broadening, compared to the intrinsically narrow features observed in SLSNe-I (Gal-Yam 2019a; Quimby et al. 2018).

addition, there is a contribution from Si IV $4 \mathrm{~s} 2 \mathrm{~S} \leftrightarrow 4 \mathrm{p} 2 \mathrm{P}^{\circ}$ (4090, $4117 \AA$ ) near the blue side of the "W" feature, which produces a distinct feature in models with lower velocities and which could explain the observed feature on the blue side of the "W" feature.

In spite of the overall good agreement, there are also some differences between the model and the observations. In particular the model spectrum is bluer and the velocities are higher. These two quantities are in tension and a better fit to one of them would result in a worse fit to the other. As mentioned above, the ionization level might be too high in the model, which suggests that the temperature might be too high as well. It should be noted that adding host extinction (which is assumed to be zero) or reducing the distance (within the error bars) would help in making

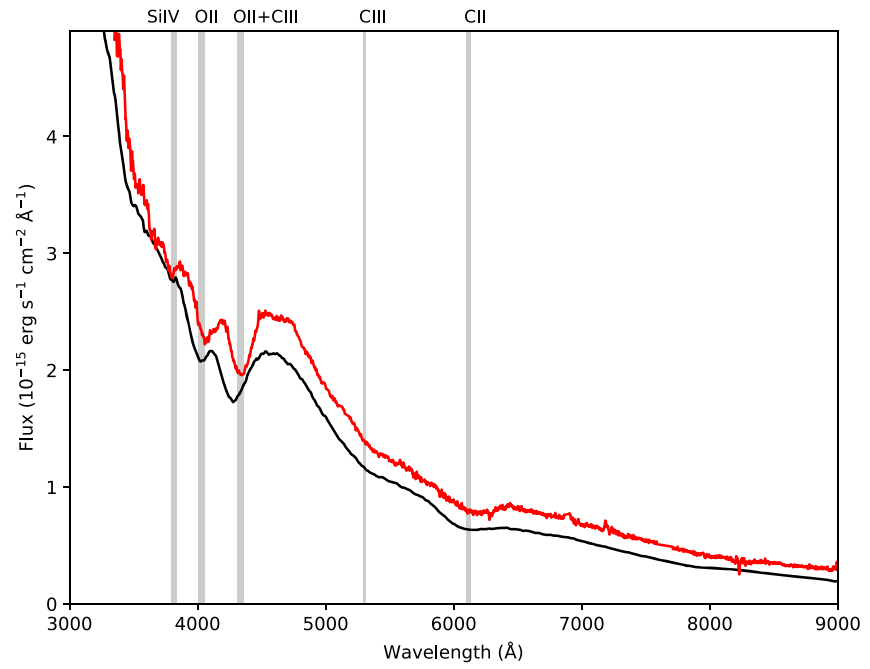

Figure 12. Observed spectrum (red) at 4.2 days, compared to our model spectrum (black) from the spectral synthesis code JEKYLL configured to run in steady state using a full NLTE solution. The model has a $\mathrm{C} / \mathrm{O}$ composition, an inner border at $22,000 \mathrm{~km} \mathrm{~s}^{-1}$, a density of $4 \times 10^{-12} \mathrm{~g} \mathrm{~cm}^{-3}$, and a density profile with a powerlaw index of -9 . The absolute (but not relative) flux of the spectrum was calibrated using the interpolated P48 $g$ and $r$ magnitudes. We also show the O II, C II, C III, and Si IV lines discussed in the text shifted to the velocity of the model photosphere.

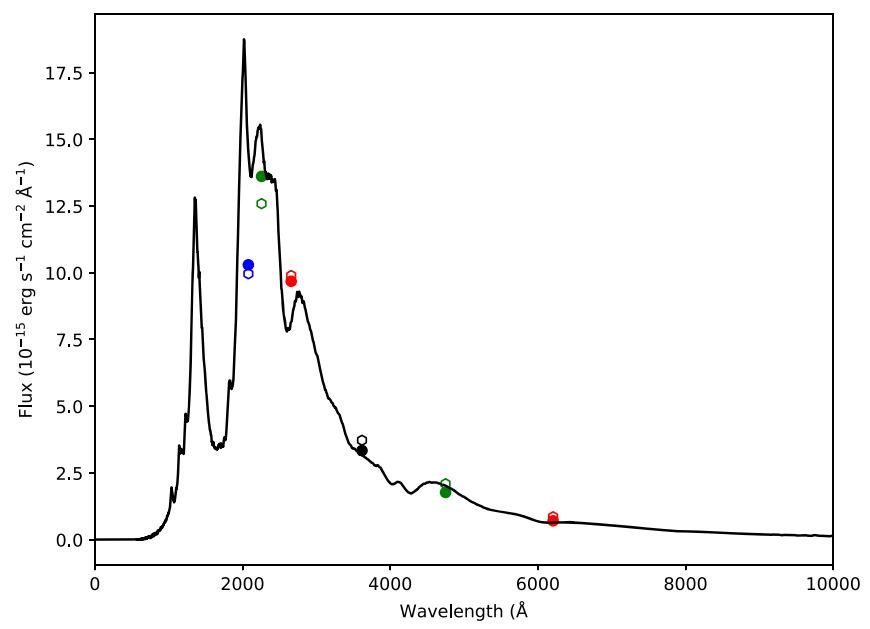

Figure 13. Comparison of model (filled circles) and observed (unfilled circles) mean fluxes through the Swift UVW1 (blue), UVM2 (green), UVW2 (red), and the SDSS $u$ (black), $g$ (green), and $r$ (red) filters. We also show the model spectrum in black.

the model redder (in the observer frame), and the latter would also help in reducing the temperature. The (modest) differences between the model and the observations could also be related to physics not included in the model, like a nonhomologous velocity field, departures from spherical asymmetry, and clumping.

The total luminosity of the model is $6.2 \times 10^{43} \mathrm{erg} \mathrm{s}^{-1}$, the photosphere is located at $\sim 33,000 \mathrm{~km} \mathrm{~s}^{-1}$, and the temperature at the photosphere is $\sim 17,500 \mathrm{~K}$, which is consistent with the values estimated from the blackbody fits (although the blackbody radius and temperature fits refer to the thermalization layer). As mentioned, we have also tried models with an $\mathrm{O} / \mathrm{Ne} /$ $\mathrm{Mg}$ composition. However, these models failed to reproduce the carbon lines redwards of the " $\mathrm{W}$ " feature. We therefore conclude that the (outer) ejecta probably have a C/O-like composition, and that this composition in combination with a standard powerlaw density profile reproduces the spectrum of SN2018gep at the 


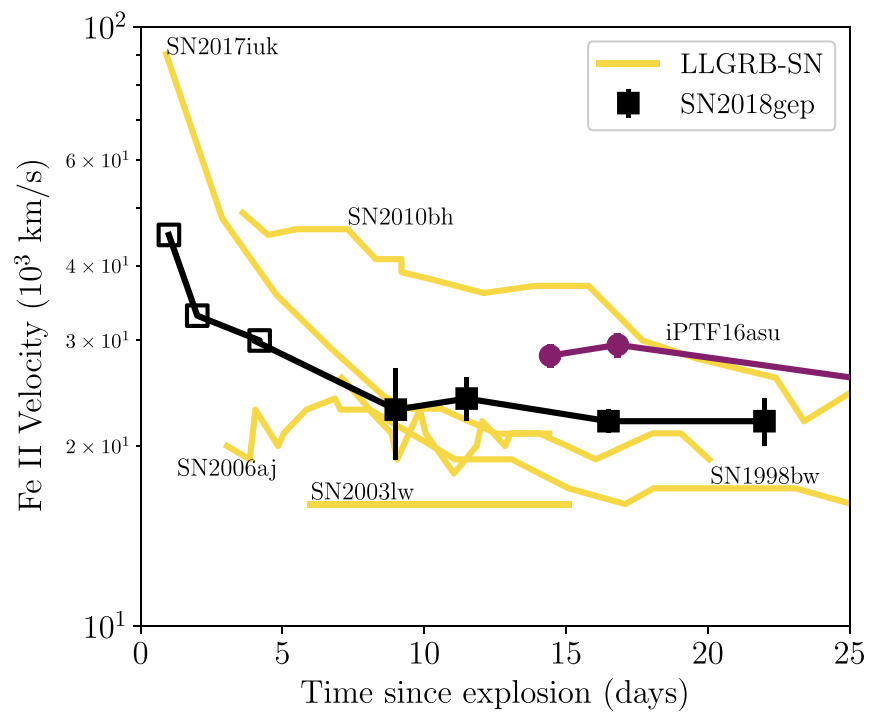

Figure 14. Velocity evolution over time as measured from spectral absorption features. Open symbols for SN2018gep come from C/O velocities measured from line minima. Closed symbols come from the Fe II feature in the Ic-BL spectra. The velocities are comparable to those measured for Ic-BL SNe associated with lowluminosity GRBs (LLGRBs). The velocity evolution for SN2017iuk is taken from Izzo et al. (2019). Velocities for iPTF16asu are taken from Whitesides et al (2017). Velocities for the other Ic-BL SNe are taken from Modjaz et al. (2016) and shifted from $V$-band max using data from Galama et al. (1998), Campana et al. (2006), Malesani et al. (2004), and Bufano et al. (2012).

observed conditions (luminosity and velocity) 4.2 days after explosion.

In our model, the broad feature seen in our Swift UVOT grism spectrum is dominated by the strong $\mathrm{Mg}$ II (2796, $2803 \AA$ ) resonance line. However, a direct comparison is not reliable because the ionization is probably lower at this epoch than what we consider for our model.

\subsubsection{Photospheric Velocity from Ic-BL Spectra}

At $\Delta t \gtrsim 7.8$ days, the spectra of SN2018gep qualitatively resemble those of a stripped-envelope $\mathrm{SN}$. We measure velocities using the method in Modjaz et al. (2016), which accommodates blending of the Fe II $\lambda 5169$ line (which has been shown to be a good tracer of photospheric velocity; Branch et al. 2002) with the nearby Fe II $\lambda \lambda 4924,5018$ lines.

At earlier times, when the spectra do not resemble typical Ic$\mathrm{BL} \mathrm{SNe}$, we use our line identifications of ionized $\mathrm{C}$ and $\mathrm{O}$ to measure velocities. As shown in Figure 14, the velocity evolution we measure is comparable to that seen in Ic-BL SNe associated with GRBs (more precisely, low-luminosity GRBs; LLGRBs) which are systematically higher than those of Ic-BL $\mathrm{SNe}$ lacking GRBs (Modjaz et al. 2016). However, as discussed in Section 2.7, no GRB was detected.

\subsection{Properties of the Host Galaxy}

We infer a star formation rate of $0.09 \pm 0.01 M_{\odot} \mathrm{yr}^{-1}$ from the $\mathrm{H} \alpha$ emission line using the Kennicutt (1998) relation converted to use a Chabrier initial mass function (IMF); (Chabrier 2003; Madau \& Dickinson 2014). We note that this is a lower limit as the slit of the Keck observation did not enclose the entire galaxy. We estimate a correction factor of 2-3: the slit diameter in the Keck spectra was $1 . " 0$, and the extraction radius was $\sim 1$ !' 75 in the February observation and $\sim 1.21^{\prime \prime}$ in the March observation. The host diameter is roughly $4^{\prime \prime}$.
We derive an electron temperature of $13,100_{-1000}^{+900} \mathrm{~K}$ from the flux ratio between [O III] $\lambda 4641$ and [O III] $\lambda 5007$, using the software package PYNEB version 1.1.7 (Luridiana et al. 2015). In combination with the flux measurements of [O II] $\lambda \lambda 3226$, 3729, [O III] $\lambda 4364$, [O III] $\lambda 4960$, [O III] $\lambda 5008$, and $\mathrm{H} \beta$, we infer a total oxygen abundance of $8.01_{-0.09}^{+0.10}$ (statistical error; using Equations (3) and (5) in Izotov et al. 2006). Assuming a solar abundance of 8.69 (Asplund et al. 2009), the metallicity of the host is $\sim 20 \%$ solar.

We also compute the oxygen abundance using the strongline metallicity indicator O3N2 (Pettini \& Pagel 2004) with the updated calibration reported in Marino et al. (2013). The oxygen abundance in the $\mathrm{O} 3 \mathrm{~N} 2$ scale is $8.05 \pm 0.01$ (stat) \pm 0.10 (sys). ${ }^{39}$

We also estimate mass and star formation rate by modeling the host SED; see Appendix D for a table of measurements, and details on where we obtained them. We use the software package LEPHARE version 2.2 (Arnouts et al. 1999; Ilbert et al.

2006). We generated $3.9 \times 10^{6}$ templates based on the Bruzual \& Charlot (2003) stellar population synthesis models with the Chabrier IMF (Chabrier 2003). The star formation history (SFH) was approximated by a declining exponential function of the form $\exp (t / \tau)$, where $t$ is the age of the stellar population and $\tau$ the e-folding timescale of the SFH (varied in nine steps between 0.1 and $30 \mathrm{Gyr}$ ). These templates were attenuated with the Calzetti attenuation curve (Calzetti et al. 2000) varied in 22 steps from $E(B-V)=0$ to $1 \mathrm{mag}$.

As shown in Figure 15, the SED is well characterized by a galaxy mass of $\log M / M_{\odot}=8.11_{-0.08}^{+0.07}$ and an attenuationcorrected star formation rate of $0.12_{-0.05}^{+0.08} M_{\odot} \mathrm{yr}^{-1}$. The derived star formation rate is comparable to the measurement inferred from $\mathrm{H} \alpha$. The attenuation of the SED is marginal, with $E(B-V)_{\text {star }}=0.05$, and consistent with the negligible Balmer decrement (Section 2.8).

Figure 16 shows that the host galaxy of SN2018gep is even more low-mass and metal-poor than the typical host galaxies of Ic$\mathrm{BL} \mathrm{SNe}$, which are low-mass and metal-poor compared to the overall core-collapse SN population. The figure uses data for 28 IcBL SNe from PTF and iPTF (Modjaz et al. 2019; Taddia et al. 2019) and a sample of 11 long-duration GRBs (including LLGRBs, all at $z<0.3$ ). We measured the emission lines from the spectra presented in Taddia et al. (2019) and used line measurements reported in Modjaz et al. (2019) for objects with missing line fluxes. The photometry was taken from S. Schulze et al. (2019, in preparation). Photometry and spectroscopy were taken from a variety of sources. ${ }^{40}$ The oxygen abundances were measured in the O3N2 scale like for SN2018gep and their SEDs were modeled with the same set of galaxy templates. For reference, the mass and SFR of the host of AT2018cow was $1.4 \times 10^{9} \mathrm{M}_{\odot}$ and $0.22 M_{\odot} \mathrm{yr}^{-1}$, respectively (Perley et al. 2019). The mass and SFR of the host of iPTF16asu was $4.6_{-2.3}^{+6.5} \times 10^{8} M_{\odot}$ and $0.7 M_{\odot} \mathrm{yr}^{-1}$, respectively (Whitesides et al. 2017).

\footnotetext{
${ }^{39}$ Note, the oxygen abundance of SN2018gep's host lies outside of the domain calibrated by Marino et al. (2013). However, we will use the measurement from the $\mathrm{O} 3 \mathrm{~N} 2$ indicator only to put the host in context of other galaxy samples that are on average more metal enriched.

${ }^{40}$ Gorosabel et al. (2005), Bersier et al. (2006), Margutti et al. (2007), Ovaldsen et al. (2007), Kocevski et al. (2007), Thöne et al. (2008), Michałowski et al. (2009), Han et al. (2010), Levesque et al. (2010), Starling et al. (2011), Hjorth et al. (2012), Thöne et al. (2014), Schulze et al. (2014), Krühler et al. (2015), Stanway et al. (2015), Toy et al. (2016), Izzo et al. (2017), and Cano et al. (2017).
} 


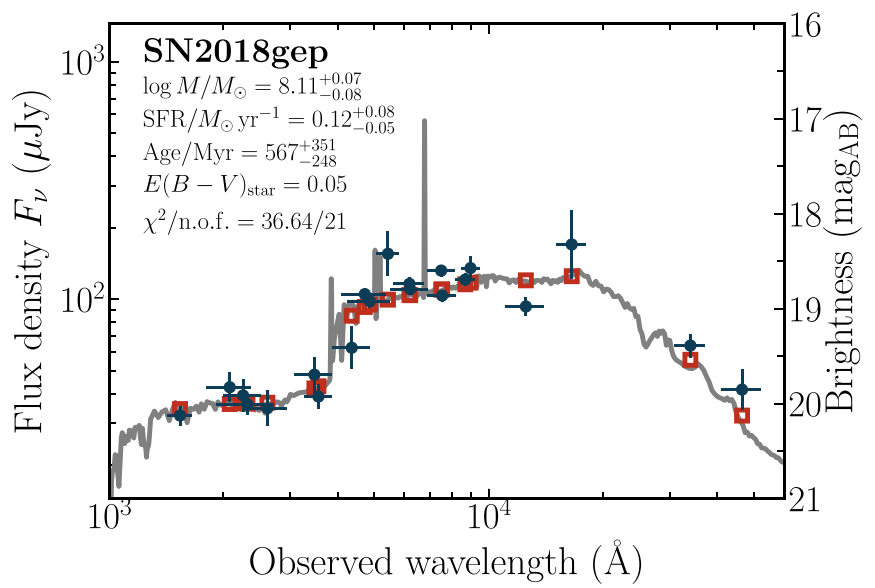

Figure 15. The spectral energy distribution of the host galaxy of SN2018gep from 1000 to $60000 \AA$ and the best fit (solid line) in the observer frame. Filled data points represent photometric measurements. The error bars in the " $x$ " direction indicate the FWHM of each filter response function. The open data points signify the model-predicted magnitudes. The quoted values of the host properties represent the median values and the corresponding $1 \sigma$ errors.

\section{Interpretation}

In Sections 2 and 3, we presented our observations and basic inferred properties of SN2018gep and its host galaxy. Now we consider what we can learn about the progenitor, beginning with the power source for the light curve.

\subsection{Radioactive Decay}

The majority of stripped-envelope $\mathrm{SNe}$ have light curves powered by the radioactive decay of ${ }^{56} \mathrm{Ni}$. As discussed in Kasen (2017), this mechanism can be ruled out for light curves that rise rapidly to a high peak luminosity, because this would require the unphysical condition of a nickel mass that exceeds the total ejecta mass. With a peak luminosity exceeding $10^{44} \mathrm{erg} \mathrm{s}^{-1}$ and a rise to peak of a few days, SN2018gep clearly falls into the disallowed region (see Figure 1 in Kasen 2017). Thus, we rule out radioactive decay as the mechanism powering the peak of the light curve.

We now consider whether radioactive decay could dominate the light curve at late times $\left(t \gg t_{\text {peak }}\right)$. The left panel of Figure 17 shows the bolometric light curve of SN2018gep compared to several other Ic-BL SNe from the literature (Cano 2013), whose light curves are thought to be dominated by the radioactive decay of ${ }^{56} \mathrm{Ni}$ (although see Moriya et al. 2017 for another possible interpretation). The luminosity of SN2018gep at $t \sim 20$ days is about half that of SN1998bw, and double that of SN2010bh and SN2006aj. By modeling the light curves of the three Ic-BL SNe shown, Cano (2013) infers nickel masses of $0.42 M_{\odot}, 0.12 M_{\odot}$, and $0.21 M_{\odot}$, respectively. On this scale, SN2018gep has $M_{\mathrm{Ni}} \sim 0.1-0.2 M_{\odot}$.

The right panel of Figure 17 shows the light curve of SN2018gep compared to that of AT2018cow (Perley et al. 2019). To estimate the nickel mass of AT2018cow, Perley et al. (2019) compared the bolometric luminosity at $t \sim 20$ days to that of SN2002ap (whose nickel mass was derived via late-time nebular spectroscopy; Foley et al. 2003) and found $M_{\mathrm{Ni}}<0.05 M_{\odot}$. On this scale, we would expect $M_{\mathrm{Ni}} \lesssim 0.05 M_{\odot}$ for SN2018gep as well.

Finally, Katz et al. (2013) and Wygoda et al. (2019) present an analytical technique for testing whether a light curve is powered by radioactive decay. At late times, the bolometric luminosity is equal to the rate of energy deposition by
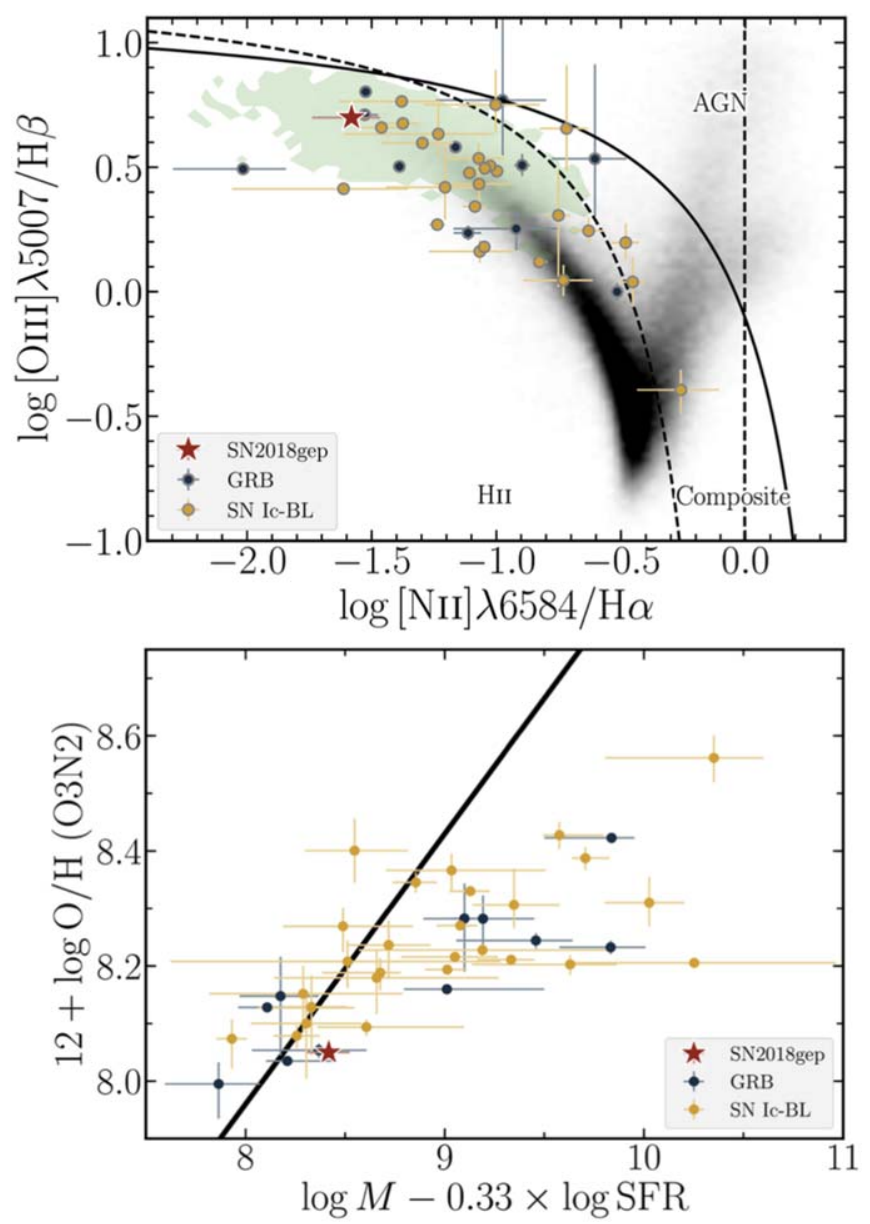

Figure 16. Top: BPT diagram. The host of SN2018gep is a low-metallicity galaxy with an intense ionizing radiation field (green shaded region indicates extreme emission line galaxies). The majority of Ic-BL SNe and long-duration GRBs are found in more metal enriched galaxies (parameterized by $[\mathrm{N} \mathrm{II}] / \mathrm{H} \alpha$ ), and galaxies with less intense radiation fields (parameterized by $[\mathrm{O} \mathrm{III]} / \mathrm{H} \alpha$ ). Field galaxies from SDSS DR15 are shown as a background density distribution. The thick solid line separates star formation- and AGN-dominated galaxies (Kewley et al. 2001). The thick dashed lines encircle the region of composite galaxies (Kauffmann et al. 2003). Bottom: the mass-metallicity-star formation-rate plane. The bulk of the SN-Ic-BL and GRB host populations are found in hosts that are more metal enriched. For reference, the host of AT2018cow had $\log M-0.33 \times \log \mathrm{SFR} \approx 9.4$. The black line is the fundamental metallicity relation in Mannucci et al. (2010).

radioactive decay $Q(t)$, because the diffusion time is much shorter than the dynamical time: $L_{\mathrm{bol}}(t)=Q(t)$. At any given time, the energy deposition rate $Q(t)$ is

$$
Q(t)=Q_{\gamma}(t)\left(1-e^{-\left(t_{0} / t\right)^{2}}\right)+Q_{\mathrm{pos}}(t)
$$

where $Q_{\gamma}(t)$ is the energy release rate of gamma-rays and $t_{0}$ is the time at which the ejecta becomes optically thin to gammarays. The expression for $Q_{\gamma}(t)$ is

$$
\frac{Q_{\gamma}(t)}{10^{43} \mathrm{erg} \mathrm{s}^{-1}}=\frac{M_{\mathrm{Ni}}}{M_{\odot}}\left(6.45 e^{-t / 8.76 \text { days }}+1.38 e^{-t / 111.4 \text { days }}\right) \text {. }
$$

$Q_{\mathrm{pos}}(t)$ is the energy deposition rate of positron kinetic energy, and the expression is

$$
\frac{Q_{\mathrm{pos}}(t)}{10^{41} \mathrm{erg} \mathrm{s}^{-1}}=4.64 \frac{M_{\mathrm{Ni}}}{M_{\odot}}\left(-e^{-t / 8.76 \text { days }}+e^{-t / 111.4 \text { days }}\right) \text {. }
$$

The dotted line in Figure 17 shows a model track with $M_{\mathrm{Ni}}=0.28 M_{\odot}$ and $t_{0}=30$ days. Lower nickel masses produce tracks that are too low to reproduce the data, and 


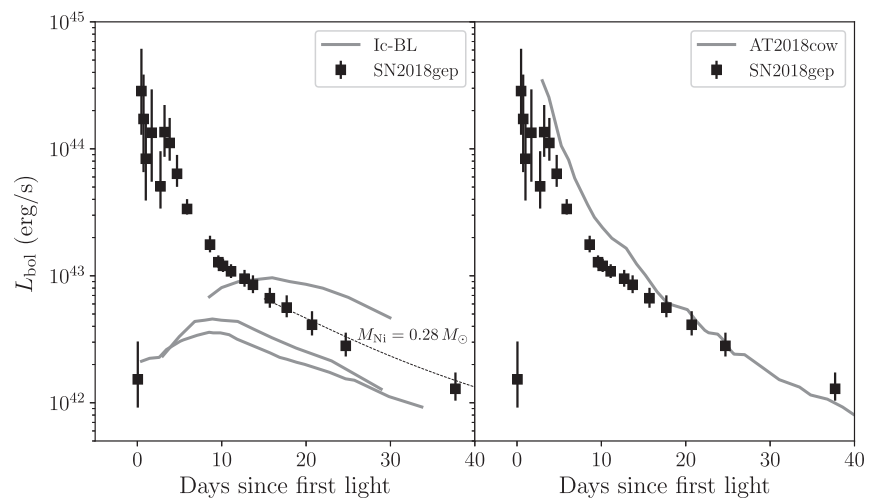

Figure 17. The bolometric light curve of SN2018gep compared to (left) other Ic-BL SNe from the literature (Cano 2013) and (right) to AT2018cow (Perley et al. 2019). The dotted line shows the expected contribution from the radioactive decay of ${ }^{56} \mathrm{Ni}$, for a gamma-ray escape time of 30 days and $M_{\mathrm{Ni}}=0.28 M_{\odot}$. In order of decreasing $L_{\mathrm{bol}}$, the three Ic-BL $\mathrm{SNe}$ are SN1998bw, SN2010bh, and SN2006aj.

larger values of $t_{0}$ produce tracks that drop off too rapidly. Thus on this scale it seems that $M_{\mathrm{Ni}} \sim 0.3 M_{\odot}$, similar to other Ic-BL SNe (Lyman et al. 2016). Because the data have not yet converged to model tracks, we cannot solve directly for $t_{0}$ and $M_{\mathrm{Ni}}$ using the technique for Ia SNe in Wygoda et al. (2019).

We can also try to solve directly for $t_{0}$ and $M_{\mathrm{Ni}}$ using the technique for Ia SNe in Wygoda et al. (2019). The first step is to solve for $t_{0}$ using Equation (1) and a second equation resulting from the fact that the expansion is adiabatic,

$$
\int_{0}^{t} Q\left(t^{\prime}\right) t^{\prime} d t^{\prime}=\int_{0}^{t} L_{\mathrm{bol}}\left(t^{\prime}\right) t^{\prime} d t^{\prime}
$$

The ratio of Equation (1) to Equation (4) removes the dependence on $M_{\mathrm{Ni}}$, and enables $t_{0}$ to be measured. However, as shown in Figure 18, the data have not yet converged to model tracks.

\subsection{Interaction with Extended Material}

One way to power a rapid and luminous light curve is to deposit energy into CSM at large radii (Nakar \& Sari 2010; Nakar $\&$ Piro 2014; Piro 2015). Since this is a Ic-BL SN, we expect the progenitor to be stripped of its envelope and therefore compact ( $R \sim 0.5 R_{\odot} \sim 10^{10} \mathrm{~cm}$; Groh et al. 2013), although there have never been any direct progenitor detections for a Ic-BL SN.

With this expectation, extended material at larger radii would have to arise from mass loss. This would not be surprising, as massive stars are known to shed a significant fraction of their mass in winds and eruptive episodes; see Smith (2014) for a review.

First we perform an order-of-magnitude calculation to see whether the rise time and peak luminosity could be explained by a model in which shock interaction powers the light curve ("wind shock breakout"). Assuming that the progenitor ejected material with a velocity $v_{w}$ at a time $t$ prior to explosion, the radius of this material at any given time is

$$
\begin{aligned}
R_{\mathrm{sh}} & =R_{*}+v_{\mathrm{w}} t \\
& \approx\left(8.64 \times 10^{12} \mathrm{~cm}\right)\left(\frac{v_{w}}{1000 \mathrm{~km} \mathrm{~s}^{-1}}\right)\left(\frac{t}{\text { day }}\right) .
\end{aligned}
$$

For material ejected 15 days prior to explosion, traveling at $1000 \mathrm{~km} \mathrm{~s}^{-1}$, the radius would be $R_{\mathrm{CSM}} \sim 10^{14} \mathrm{~cm}$ at the time

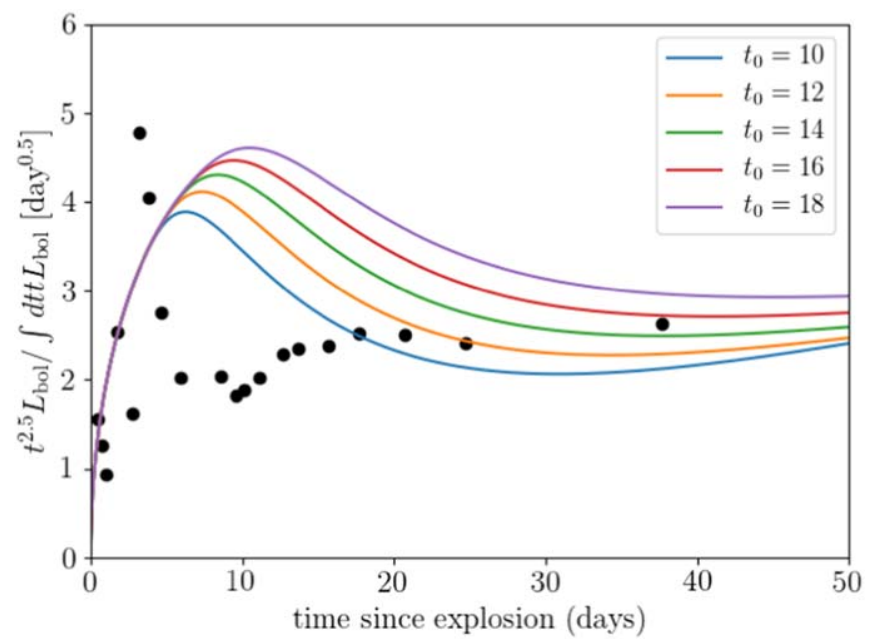

Figure 18. To test whether a light curve is powered by radioactive decay, the ratio of the bolometric luminosity to the time-weighted integrated bolometric luminosity should converge to model tracks, as described in Katz et al. (2013) and Wygoda et al. (2019). This enables a direct measurement of the gamma-ray escape time $t_{0}$ and the nickel mass $M_{\mathrm{Ni}}$. However, our data have not converged to these tracks, suggesting that either radioactive decay is not dominant, or that we are not yet in a phase where we can perform this measurement.

of explosion. The shock crossing timescale is $t_{\text {cross }}$ :

$$
t_{\text {cross }} \sim R_{\mathrm{CSM}} / v_{s} \approx(0.4 \text { day })\left(\frac{R}{10^{14} \mathrm{~cm}}\right)\left(\frac{v_{s}}{0.1 c}\right)^{-1}
$$

where $v_{s}$ is the velocity of the shock. The shock heats the CSM with an energy density that is roughly half of the kinetic energy of the shock, so $e_{s} \sim(1 / 2)\left(\rho v_{s}^{2} / 2\right)$. The luminosity is the total energy deposited divided by $t_{\text {cross }}$,

$$
\begin{aligned}
L_{\mathrm{BO}} & \sim \frac{E_{\mathrm{BO}}}{t_{\mathrm{cross}}} \sim \frac{v_{s}^{3}}{4} \frac{d M}{d R} \\
& =\left(8 \times 10^{44} \mathrm{erg} \mathrm{s}^{-1}\right)\left(\frac{v_{s}}{0.1 c}\right)^{3}\left(\frac{d M}{M_{\odot}}\right)\left(\frac{d R}{10^{14} \mathrm{~cm}}\right)^{-1}
\end{aligned}
$$

assuming a constant density. Thus, for shock velocities on the order of the observed photospheric radius expansion $(0.1 c)$, and a CSM radius on the order of the first photospheric radius that we measure $\left(3 \times 10^{14} \mathrm{~cm}\right)$, it is easy to explain the rise time and peak luminosity that we observe.

To test whether shock breakout (and subsequent post-shock cooling) can explain the evolution of the physical properties we measured in Section 3, we ran one-dimensional numerical radiation hydrodynamics simulations of an SN running into a circumstellar shell with CASTRO (Almgren et al. 2010; Zhang et al. 2011). We assume spherical symmetry and solve the coupled equations of radiation hydrodynamics using a gray flux-limited nonequilibrium diffusion approximation. The setup is similar to the models presented in Rest et al. (2018) but with parameters modified to fit SN2018gep.

The ejecta is assumed to be homologously expanding, characterized by a broken power-law density profile, an ejecta mass $M_{\mathrm{ej}}$, and energy $E_{\mathrm{ej}}$. The ejecta density profile has an inner power-law index of $n=0$ (that is, $\rho(r) \propto r^{-n}$ ) then steepens to an index $n=10$, as is appropriate for core collapse SN explosions (Matzner \& McKee 1999). The circumstellar shell is assumed to be uniform in density with radius $R_{\mathrm{CSM}}$ and mass $M_{\mathrm{CSM}}$. We adopt a uniform opacity of $\kappa=0.2 \mathrm{~cm}^{2} \mathrm{~g}^{-1}$, which is characteristic of hydrogen-poor electron scattering. 
The best-fit model, shown in Figure 19, used the following parameters: $M_{\mathrm{ej}}=8 M_{\odot}, E_{\mathrm{ej}}=2 \times 10^{52} \mathrm{erg}, M_{\mathrm{CSM}}=0.02 M_{\odot}$, and $R_{\mathrm{CSM}}=3 \times 10^{14} \mathrm{~cm}$. The inferred kinetic energy is consistent with typical values measured for Ic-BL SNe (e.g., Cano et al. 2017; Taddia et al. 2019), and $R_{\mathrm{CSM}}$ is similar in value to the first photospheric radius we measure (at $\Delta t=0.05$ day; see Figure 9).

The inferred values presented here are likely uncertain to within a factor of a few, given the degeneracies of the rise time and peak luminosity with the CSM mass and radius. Qualitatively, a larger CSM radius will result in a higher peak luminosity and longer rise time. The peak luminosity is relatively independent of the CSM mass, which instead affects the photospheric velocity and temperature (i.e., a larger CSM mass slows down the post-interaction velocity to a greater extent and increases the shock-heated temperature). A full discussion of the dependencies of the light curve and photospheric properties on the CSM parameters will be presented in an upcoming work (D. Khatami et al. 2019, in preparation.).

In this framework, the shockwave sweeps through the CSM prior to peak luminosity, so that at maximum luminosity the outer parts of the CSM have been swept into a dense shell moving at SN-like velocities $\left(v_{\text {post-shock }} \approx 3 v_{s} / 4\right)$. This scenario was laid out in Chevalier \& Irwin (2011) and discussed in Kasen (2017). This explains the high velocities we measure at early times and the absence of narrow emission features in our spectra. For another discussion of the absence of narrow emission lines due to an abrupt cutoff in CSM density, see Moriya \& Tominaga (2012). Following Chevalier \& Irwin (2011), the rapid rise corresponds to shock breakout from the CSM, and begins at a time $R_{\mathrm{CSM}} / v_{\mathrm{sh}}$ after the explosion, where $v_{\mathrm{sh}}$ is the velocity of the shock. The time to peak luminosity ( 1.2 days) is longer than this delay time by a factor $\left(R_{w} / R_{d}\right)$. Given the best-fit $R_{w}=3 \times$ $10^{14} \mathrm{~cm}$, and assuming $R_{d} \sim R_{w}$, we find $v_{\mathrm{sh}}=0.1 c$, and an explosion time $\sim 1$ day prior to $t_{0}$. This model also predicts an increasing temperature while the shock breaks out (i.e., during the rise to peak bolometric luminosity).

Other Ic SNe have shown early evidence for interaction in their light curves, but in other cases the emission has been attributed to post-shock cooling in expanding material rather than shock breakout itself. For example, the first peak observed in iPTF14gqr (De et al. 2018) was short-lived ( $\lesssim 2$ days) and attributed to shock-cooling emission from material stripped by a compact companion. iPTF14gqr is different in a number of ways from SN2018gep: the spectra showed high-ionization emission lines, including He II, and the explosion had a much smaller kinetic energy $\left(E_{K} \approx 10^{50} \mathrm{erg}\right)$ and smaller velocities $\left(10,000 \mathrm{~km} \mathrm{~s}^{-1}\right)$. The main peak in iPTF16asu was also modeled as shock-cooling emission rather than shock breakout (Whitesides et al. 2017).

Under the assumption that the light curve represented postshock cooling emission, De et al. (2018) and Whitesides et al. (2017) both used one-zone analytic models from Piro (2015) to estimate the properties of the explosion and the CSM. This approximation assumes that the emitting region is a uniformly heated expanding sphere. In iPTF14gqr the inferred properties of the extended material were $M_{e} \sim 8 \times 10^{-3} M_{\odot}$ at $R_{e} \sim 3 \times$ $10^{13} \mathrm{~cm}$. In iPTF16asu the inferred properties of the extended material were $M_{e} \sim 0.45 M_{\odot}$ at $R_{e} \sim 1.7 \times 10^{12} \mathrm{~cm}$. The fit also required a more energetic explosion than iPTF14gqr $\left(4 \times 10^{51} \mathrm{erg}\right)$. By applying the same framework to the decline of the bolometric light curve of SN2018gep, we arrive at similar values to those inferred for iPTF16asu, as shown in Figure 20.
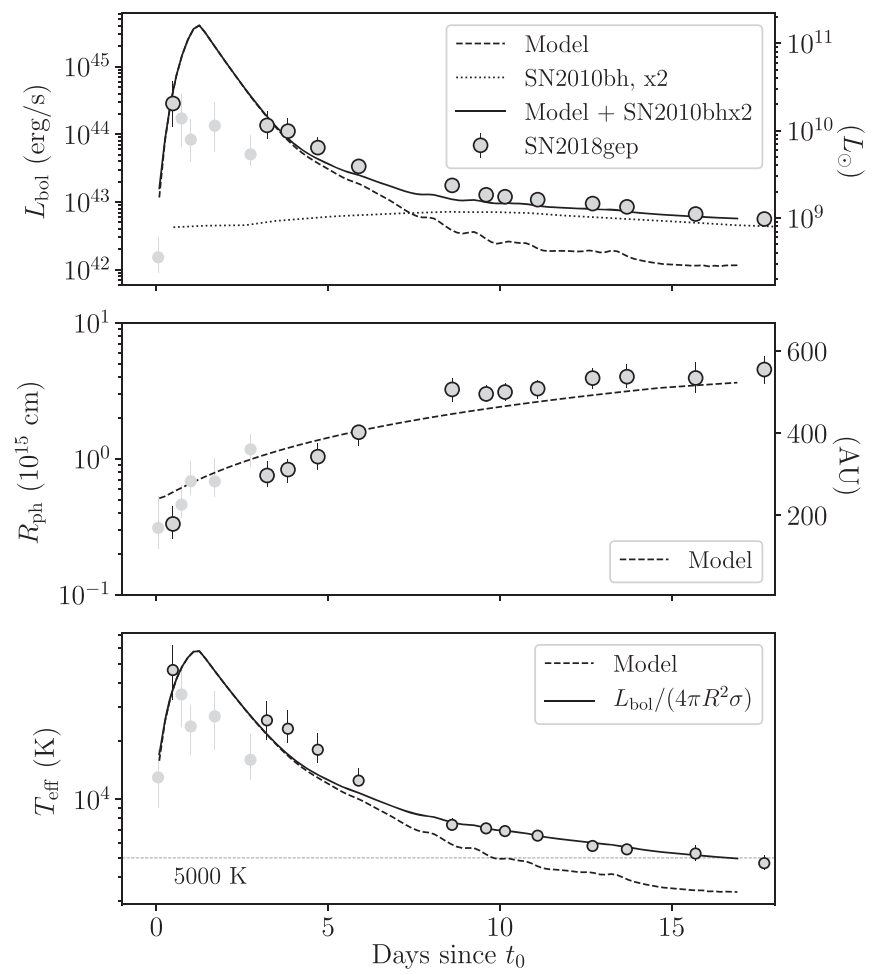

Figure 19. Best-fit CSM interaction model with the light curve of the Ic-BL SN 2010bh (Cano 2013) scaled up by a factor of two. The model parameters are $M_{\mathrm{ej}}=8 M_{\odot}, \quad E_{\mathrm{ej}}=2 \times 10^{52} \mathrm{erg}, \quad M_{\mathrm{CSM}}=0.02 M_{\odot}$, and $R_{\mathrm{CSM}}=3 \times$ $10^{14} \mathrm{~cm}$. As in Figure 9, the outlined circles are derived from UV and optical data, while the light gray circles are derived from optical data only.

We model the main peak of SN2018gep as shock breakout rather than post-shock cooling emission. Our motivation for this choice is that the timescale over which we detect the precursor emission is more consistent with a large radius and lower shell mass. From the shell mass and radius, we can also estimate the mass-loss rate immediately prior to explosion,

$$
\frac{\dot{M}}{M_{\odot} \mathrm{yr}^{-1}} \approx 32\left(\frac{M_{\mathrm{sh}}}{M_{\odot}}\right)\left(\frac{v_{w}}{1000 \mathrm{~km} \mathrm{~s}^{-1}}\right)\left(\frac{R_{\mathrm{sh}}}{10^{14} \mathrm{~cm}}\right)^{-1} .
$$

For our best-fit parameters $M_{\mathrm{sh}}=0.02 M_{\odot}$ and $R_{\mathrm{sh}}=3 \times$ $10^{14} \mathrm{~cm}$, and taking $v_{w}=1000 \mathrm{~km} \mathrm{~s}^{-1}$, we find $\dot{M} \approx$ $0.6 M_{\odot} \mathrm{yr}^{-1}$, 4-6 orders of magnitude higher than what is typically expected for Ic-BL SNe (Smith 2014).

In the shock breakout model, the shock sweeps through confined CSM and passes into lower density material. Thus, it is not surprising that we do not observe the X-ray or radio emission that would indicate interaction with high-density material. From our VLA observations of SN2018gep, the radio flux marginally decreased from $\Delta t=5$ days to $\Delta t=75$ days. This could be astrophysical, but could also be instrumental (change in beam size due to change in VLA configuration). Using the relation of Murphy et al. (2011), the estimated contribution from the host galaxy (for an SFR of $0.12_{-0.05}^{+0.08} M_{\odot} \mathrm{yr}^{-1}$; see Section 3.3) is

$$
\begin{aligned}
\left(\frac{L_{1.4 \mathrm{GHz}}}{\mathrm{erg} \mathrm{s}^{-1} \mathrm{~Hz}^{-1}}\right) & \approx 1.57 \times 10^{28}\left(\frac{\mathrm{SFR}_{\text {radio }}}{M_{\odot} \mathrm{yr}^{-1}}\right) \\
& \approx 1.9 \times 10^{27} \mathrm{erg} \mathrm{s}^{-1} \mathrm{~Hz}^{-1} .
\end{aligned}
$$

Taking a spectral index of -0.7 (a synchrotron spectrum), the expected $9 \mathrm{GHz}$ luminosity would be between $3.0 \times$ $10^{26} \mathrm{erg} \mathrm{s}^{-1} \mathrm{~Hz}^{-1}$ and $8.6 \times 10^{26} \mathrm{erg} \mathrm{s}^{-1} \mathrm{~Hz}^{-1}$. From Table 2, 


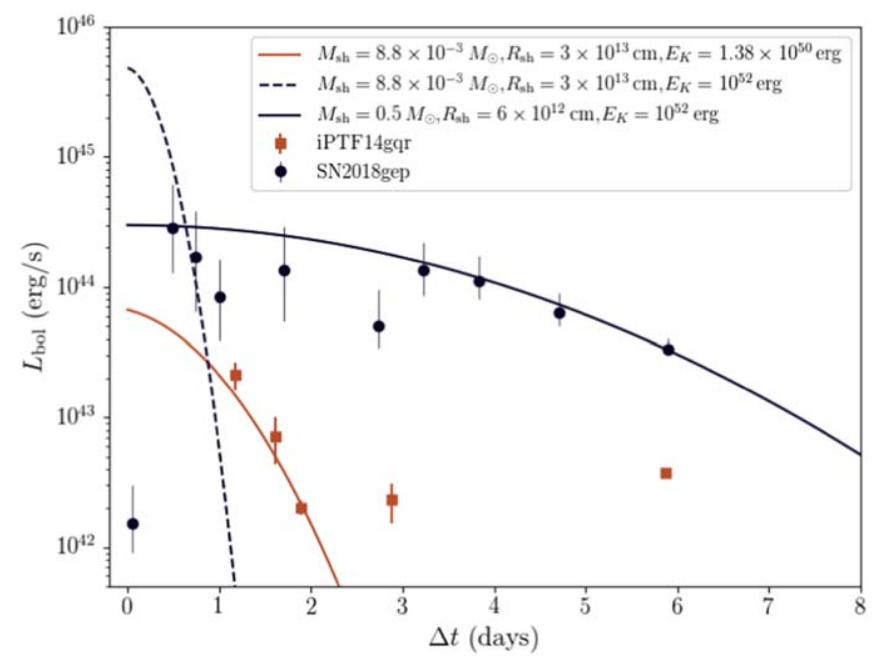

Figure 20. Estimated CSM and explosion properties using models from Piro (2015). The shell mass is much larger than the one in iPTF14gqr, which is the reason for the more extended shock-cooling peak.

the measured spectral luminosity is $8.3 \times 10^{26} \mathrm{erg} \mathrm{s}^{-1} \mathrm{~Hz}^{-1}$ (at $10 \mathrm{GHz}$ ) in the first epoch, and $6 \times 10^{26} \mathrm{erg} \mathrm{s}^{-1} \mathrm{~Hz}^{-1}$ (at $9 \mathrm{GHz}$ ) in the second epoch. The slit covering fraction of our LRIS observations is again relevant here; as discussed in Section 3.3, the true SFR is likely a factor of a few higher than what we inferred from modeling the galaxy SED. So, it is plausible that the first two radio detections are entirely due to the host galaxy.

In the third epoch, the luminosity (at $9 \mathrm{GHz}$ ) is $<3.9 \times 10^{26} \mathrm{erg} \mathrm{s}^{-1} \mathrm{~Hz}^{-1}$, although the difference from the first two epochs may be due to the different array configuration. Taking the peak of the $9-10 \mathrm{GHz}$ light curve to be $8.3 \times 10^{26} \mathrm{erg} \mathrm{s}^{-1} \mathrm{~Hz}^{-1}$ at $\Delta t \approx 5$ days, Figure 21 shows that SN2018gep would be an order of magnitude less luminous in radio emission than any other Ic-BL SN. If the luminosity truly decreased, then the implied mass-loss rate is $\dot{M} \sim 3 \times 10^{-6}$, consistent with the idea that the shock has passed from confined CSM into much lower density material.

If the emission is constant and due entirely to the host galaxy, the point shown in Figure 21 is an upper limit in luminosity. Assuming that the peak of the SED of any radio emission from the $\mathrm{SN}$ is not substantially different from the frequencies we measure (i.e., that the spectrum is not selfabsorbed at these frequencies), we have a limit on the $9 \mathrm{GHz}$ radio luminosity of $L_{p} \lesssim 10^{27} \mathrm{erg} \mathrm{s}^{-1} \mathrm{~Hz}^{-1}$ at $\Delta t \approx 5-15$ days.

The shell mass and radius also give an estimate of the optical depth: $\tau \approx \kappa M / r^{2} \approx 100>>1$, which means that the shell would be optically thick. The lack of detected X-ray emission is consistent with the expectation that any X-ray photons produced in the collision would be thermalized by the shell and reradiated as blackbody emission.

Finally, assuming that the rapid rise to peak is indeed caused by shock breakout, we examine whether our model is consistent with our detections in the weeks prior to explosion. Material ejected 10 days prior to the explosion at the escape velocity of a Wolf-Rayet star $\left(v_{\mathrm{esc}} \sim 1000 \mathrm{~km} \mathrm{~s}^{-1}\right)$ would lie at $R \sim 10^{14} \mathrm{~cm}$, which is consistent with our model. Assuming that the emission mechanism is internal shocks between shells of ejected material traveling at different velocities, we can estimate the amount of mass required:

$$
\frac{1}{2} \epsilon M v^{2}=L \tau
$$

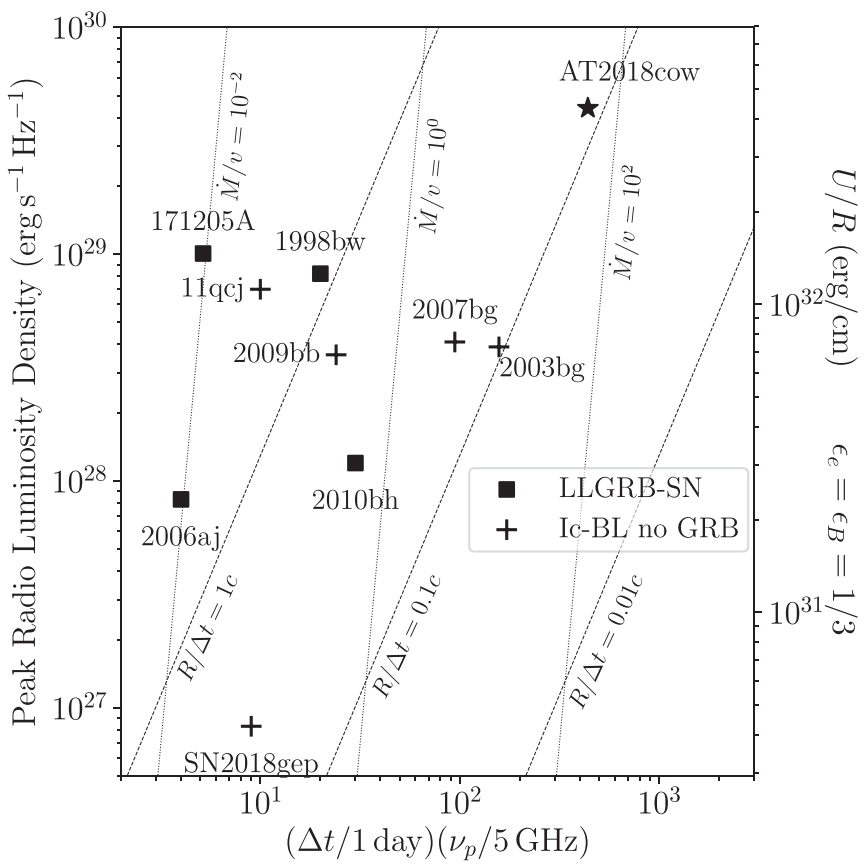

Figure 21. The radio luminosity of SN2018gep compared to AT2018cow and radio-loud Ic-BL SNe (assuming $\epsilon_{e}=\epsilon_{B}=1 / 3$, see Chevalier 1998; Soderberg et al. 2010; Ho et al. 2019). Lines of constant mass-loss rate (scaled to wind velocity) are shown in units of $10^{-4} M_{\odot} \mathrm{yr}^{-1} / 1000 \mathrm{~km} \mathrm{~s}^{-1}$. The radio luminosity for GRB 171205A was taken from VLA observations reported by Laskar et al. (2017), but we note that this is a lower limit in luminosity and in peak frequency because the source was heavily self-absorbed at this epoch.

where $v \approx 1000 \mathrm{~km} \mathrm{~s}^{-1}, \epsilon \approx 0.5$ is the efficiency of thermalizing the kinetic energy of the shells, $M$ is the shell mass, $L \approx$ $10^{39} \mathrm{erg} \mathrm{s}^{-1}$ is the luminosity we observe, and $\tau \approx 10$ days is the timescale over which we observe the emission. We find $M \approx$ $0.02 M_{\odot}$, again consistent with our model.

We conclude that the data are consistent with a scenario in which a compact Ic-BL progenitor underwent a period of eruptive mass loss shortly prior to explosion. In the terminal explosion, the light curve was initially dominated by shock breakout through (and post-shock cooling of) this recently ejected material.

Finally, we return to the question of the emission detected in the first few minutes, which showed an inflection point prior to the rapid rise to peak (Figure 2). Given the pre-explosion activity and inference of CSM interaction, it is not surprising that the rise is not well-modeled by a simple quadratic function. One possibility is that we are seeing ejecta already heated from earlier precursor activity. Another possibility is that we are seeing the effects of a finite light travel time. For a sphere of $R \sim 3 \times 10^{14} \mathrm{~cm}$, the light-crossing time is $\sim 20$ minutes. The slower rising phase could represent the time for photons to reach us across the extent of the emitting sphere.

In Table 4, we summarize the key properties inferred from Section 4.

\section{Comparison to Unclassified Rapidly Evolving Transients at High Redshift}

In terms of the timescale of its light curve evolution, SN2018gep is similar to AT2018cow in fulfilling the criteria that optical surveys use to identify rapidly evolving transients (e.g., Drout et al. 2014; Tanaka et al. 2016; Pursiainen et al. 2018). However, there are a number of ways in which SN2018gep is more of a "typical" member of these populations 
Table 4

Key Model Properties of SN2018gep

\begin{tabular}{lcc}
\hline \hline Parameter & Value & Notes \\
\hline$t_{\text {rise }}$ & 1.2 days & \\
$E_{\mathrm{SN}}$ & $2 \times 10^{52} \mathrm{erg}$ & \\
$M_{\mathrm{ej}}$ & $8 M_{\odot}$ & \\
$M_{\mathrm{CSM}}$ & $0.02 M_{\odot}$ & Assuming $v_{w}=1000 \mathrm{~km} \mathrm{~s}^{-1}$ \\
$R_{\mathrm{CSM}}$ & $3 \times 10^{14} \mathrm{~cm}$ & \\
$\dot{M}$ & $0.6 M_{\odot} \mathrm{yr}^{-1}$ & \\
$M_{\mathrm{Ni}}$ & $<0.2-0.3 M_{\odot}$ & \\
\hline
\end{tabular}

than AT2018cow. In particular, SN2018gep has an expanding photospheric radius and declining effective temperature. By contrast, one of the challenges in explaining AT2018cow as a stellar explosion was its nearly constant temperature (persistent blue color) and declining photospheric radius. In Figure 22 we show these two different kinds of evolution as very different tracks in color-magnitude space. We also show a late-time point for KSN2015K (Rest et al. 2018), which shows blue colors even after the transient had faded to half-max. The massloss rate inferred for Rest et al. (2018) was $2 \times 10^{-3} M_{\odot} \mathrm{yr}^{-1}$.

Of the PS-1 events, most appear to expand, cool, and redden with time (Drout et al. 2014). That said, there are few coeval data points in multiple filters, even in the gold sample transients. The transients are also faint; all but one lie at $z>0.1$. Of the DES sample, most also show evidence for declining temperatures and increasing radii, although three show evidence of a constant temperature and decreasing radius: $15 \mathrm{X} 3 \mathrm{mxf}$, 16X1eho, and 15C3opk. The peak bolometric luminosities for these three transients are reported as $3 \times 10^{43} \mathrm{erg} \mathrm{s}^{-1}, 9 \times 10^{43} \mathrm{erg} \mathrm{s}^{-1}$, and $5 \times 10^{43} \mathrm{erg} \mathrm{s}^{-1}$, respectively (Pursiainen et al. 2018).

To estimate a rate of Ic-BL SNe that have a light curve powered by shock breakout, we used the sample of 25 nearby $(z<0.1)$ Ic-BL SNe from PTF (Taddia et al. 2019), because these were found in an untargeted survey. Of these, we could not draw a conclusion about eight (either because the peak was not resolved or there was no multicolor photometry available around peak, or both). The remaining clearly lacked the rise time or blue colors of SN2018gep. Furthermore, SN2018gep is unique among the sample of 12 nearby $(z<0.1)$ Ic-BL SNe from ZTF discovered so far, which will be presented in a separate publication. From this, we estimate that the rate of Ic$\mathrm{BL} \mathrm{SNe}$ with a main peak dominated by shock breakout is no more than $10 \%$ of the rate of Ic-BL SNe.

\section{Summary and Future Work}

In this paper, we presented an unprecedented data set that connects late-stage eruptive mass loss in a stripped massive star to its subsequent explosion as a rapidly rising luminous transient. Here we summarize our key findings:

1. High-cadence dual-band observations with ZTF (six observations in $3 \mathrm{hr}$ ) captured a rapid rise $\left(1.4 \pm 0.1 \mathrm{mag} \mathrm{hr}^{-1}\right)$ to peak luminosity, and a corresponding increase in temperature. This rise rate is second only to that of SN 2016gkg (Bersten et al. 2018), which was attributed to shock breakout in extended material surrounding a Type IIb progenitor. However, the signal in SN2018gep is two magnitudes more luminous.

2. A retrospective search in ZTF data revealed clear detections of precursor emission in the days and months leading up to the terminal explosion. The luminosity of

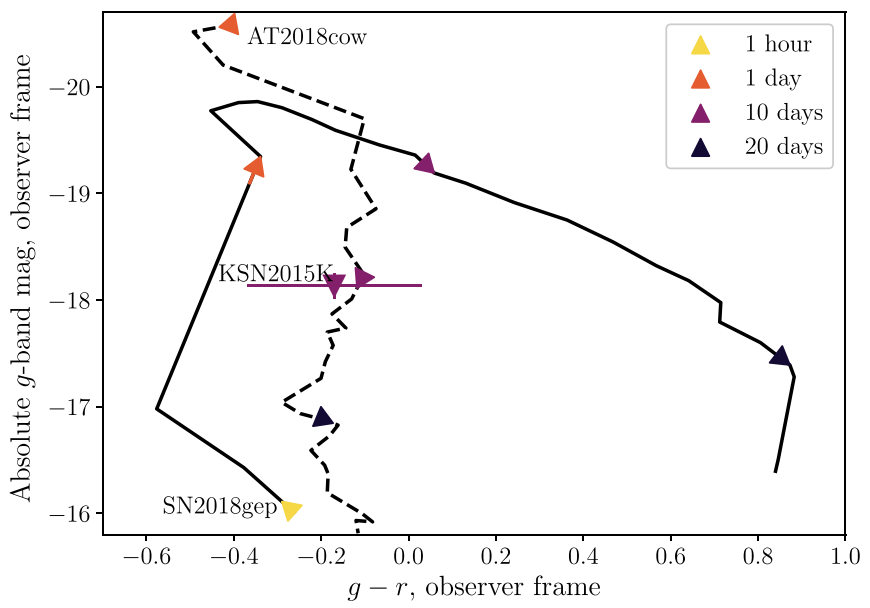

Figure 22. A "color-magnitude" diagram of AT2018cow and SN2018gep, showing the evolution of color with time from first light $\left(t_{0}\right)$. Like AT2018cow, the fast transient KSN2015K stayed persistently blue even after it had faded to half-maximum. SN2018gep has more typical SN evolution, reddening with time (cooling in temperature).

these detections $(M=-14)$ and evidence for variability suggests that they arise from eruptive mass loss, rather than the luminosity of a quiescent progenitor. This is the first definitive pre-explosion detection of a Ic-BL SN.

3. The bolometric light curve peaks after a few days at $>3 \times 10^{44} \mathrm{erg} \mathrm{s}^{-1}$. At late times, a power-law and an exponential decay are both acceptable fits to the data.

4. The temperature rises to $50,000 \mathrm{~K}$ in the first day, then declines as $t^{-1}$ then flattens at $5000 \mathrm{~K}$, which we attribute to recombination of carbon and oxygen.

5. The photosphere expands at $v=0.1 c$, and flattens once recombination sets in.

6. We obtained nine spectra in the first five days of the explosion, as the effective temperature declined from $50,000 \mathrm{~K}$ to $20,000 \mathrm{~K}$. To our knowledge, these represent the earliest-ever spectra of a stripped-envelope SN, in terms of temperature evolution.

7. The early spectra exhibit a "W" feature similar to what has been seen in stripped-envelope superluminous SNe. From an NLTE spectral synthesis model, we find that this can be reproduced with a carbon and oxygen composition.

8. The velocities inferred from the spectra are among the highest observed for stripped-envelope $\mathrm{SNe}$, and are most similar to the velocities of Ic-BL $\mathrm{SNe}$ accompanied by GRBs.

9. The host galaxy has a star formation rate of $0.12 M_{\odot} \mathrm{yr}^{-1}$, and a lower mass and lower metallicity than galaxies hosting GRB-SNe, which are low-mass and lowmetallicity compared to the overall CC SN population.

10. The early light curve is best described by shock breakout in extended but confined CSM, with $M=0.02 M_{\odot}$ at $R=3 \times 10^{14} \mathrm{~cm}$. The implied mass-loss rate is $0.6 M_{\odot} \mathrm{yr}^{-1}$ in the days leading up to the explosion, consistent with our detections of precursor emission. After the initial breakout, the shock runs through CSM of much lower density, hence the lack of narrow emission features and lack of strong radio and X-ray emission.

11. Although SN2018gep is similar to AT2018cow in terms of its bolometric light curve, it has a very different color evolution. In this sense, the "rapidly evolving transients" 
in the PS-1 and DES samples are more similar to SN2018gep than to AT2018cow.

12. The late-time light curve seems to require an energy deposition mechanism distinct from shock interaction. Radioactive decay is one possibility, but further monitoring is needed to test this.

The code used to produce the results described in this paper was written in Python and is available online in an open-source GitHub repository ${ }^{41}$ and it is archived on Zenodo (doi:10.5281/zenodo.3534067). When the paper has been accepted for publication, the data will be made publicly available via WISeREP, an interactive repository of supernova data (Yaron \& Gal-Yam 2012).

The authors would like to thank the anonymous referee whose comments improved the flow, precision, and clarity of the paper. It is a pleasure to thank Tony Piro, Dan Kasen, E. Sterl Phinney, Eliot Quataert, Maryam Modjaz, Jim Fuller, Lars Bildsten, Udi Nakar, Paul Duffell, and Luc Dessart for helpful discussions. A.Y.Q.H. is particularly grateful to Tony Piro and the community at Carnegie Observatories for their hospitality on Tuesdays during the period in which this work was performed. Thank you to the staff at the SMA, AMI, the VLA, Swift, and Chandra for rapidly scheduling and executing the observations. Thank you to David Palmer (LANL) for his assistance in searching the pointing data for Swift/ BAT. Thank you to Michael J. Koss (Eureka Scientific Int), Andrew Drake (Caltech), Scott Adams (Caltech), Matt Hankins (Caltech), Kevin Burdge (Caltech), and Kirsty Taggart (LJMU) for assisting with optical spectroscopic observations. Thank you to Erik Petigura and David Hogg for their advice on figure aesthetics. Thank you to David Alexander Kann (IAA-CSIC) for pointing out an error in an early version of the paper posted to the arXiv. D.A.G. thanks Stéfan van der Walt and Ari Crellin-Quick for assistance with skyportal, which enabled the search for pre-explosion emission.

A.Y.Q.H. is supported by a National Science Foundation Graduate Research Fellowship under grant No. DGE1144469. This work was supported by the GROWTH project funded by the National Science Foundation under PIRE grant No. 1545949. A. G.-Y. is supported by the EU via ERC grant No. 725161, the ISF, the BSF Transformative program, and by a Kimmel award. Y.T. studied as a GROWTH intern at Caltech during the summer and fall of 2017. C.C.N. thanks the funding from MOST grant 1042923-M-008-004-MY5. R.L. is supported by a Marie SkłodowskaCurie Individual Fellowship within the Horizon 2020 European Union (EU) Framework Programme for Research and Innovation (H2020-MSCA-IF-2017-794467). A.H. acknowledges support by the I-Core Program of the Planning and Budgeting Committee and the Israel Science Foundation, and support by the ISF grant 647/ 18. This research was supported by a grant from the GIF, the German-Israeli Foundation for Scientific Research and Development. This research was funded in part by the Gordon and Betty Moore Foundation through Grant GBMF5076, and a grant from the Heising-Simons Foundation. A.C. acknowledges support from the NSF CAREER award N. 1455090 and from the NASA/ Chandra GI award N. GO8-19055A. Research support to I.A. is provided by the GROWTH project, funded by the National Science Foundation under grant No 1545949.

This work is based on observations obtained with the Samuel Oschin Telescope 48 inch and the 60 inch Telescope at the Palomar

\footnotetext{
$\overline{41}$ https://github.com/annayqho/SN2018gep
}

Observatory as part of the Zwicky Transient Facility project. Major funding has been provided by the U.S. National Science Foundation under grant No. AST-1440341 and by the ZTF partner institutions: the California Institute of Technology, the Oskar Klein Centre, the Weizmann Institute of Science, the University of Maryland, the University of Washington, Deutsches ElektronenSynchrotron, the University of Wisconsin-Milwaukee, and the TANGO Program of the University System of Taiwan. Partially based on observations made with the Nordic Optical Telescope, operated by the Nordic Optical Telescope Scientific Association at the Observatorio del Roque de los Muchachos, La Palma, Spain, of the Instituto de Astrofisica de Canarias. The Liverpool Telescope is operated by Liverpool John Moores University with financial support from the UK Science and Technology Facilities Council. LT is located on the island of La Palma, in the Spanish Observatorio del Roque de los Muchachos of the Instituto de Astrofisica de Canarias. The scientific results reported in this article are based in part on observations made by the Chandra X-ray Observatory. The data presented here were obtained in part with ALFOSC, which is provided by the Instituto de Astrofisica de Andalucia (IAA) under a joint agreement with the University of Copenhagen and NOTSA. The Submillimeter Array is a joint project between the Smithsonian Astrophysical Observatory and the Academia Sinica Institute of Astronomy and Astrophysics and is funded by the Smithsonian Institution and the Academia Sinica. We acknowledge the support of the staff of the Xinglong $2.16 \mathrm{~m}$ telescope. This work is supported by the National Natural Science Foundation of China (NSFC grants 11325313 and 11633002), and the National Program on Key Research and Development Project (grant No. 2016YFA0400803). SED Machine is based upon work supported by the National Science Foundation under grant No. 1106171. This publication has made use of data collected at Lulin Observatory, partly supported by MoST grant 105-2112-M-008024-MY3. The JEKYLL simulations were performed on resources provided by the Swedish National Infrastructure for Computing (SNIC) at Parallelldatorcentrum (PDC).

Facilities: CFHT, Keck:I (LRIS), Hale (DBSP), AMI, Liverpool:2 m (IO:O, SPRAT), DCT, Swift (UVOT, XRT), Beijing:2.16 m, EVLA, SMA, LO:1 m, NOT (ALFOSC).

Software: Astropy (Astropy Collaboration et al. 2013, 2018), IPython (Pérez \& Granger 2007), matplot lib (Hunter 2007), numpy (Oliphant 2006), scipy (Jones et al. 2001), extinction (Barbary 2016) SkyPortal (Van der Walt et al. 2019).

\section{Appendix}

In Appendix A we provide the full set of optical and UV photometry and the blackbody fits to this photometry. In Appendix B we provide the log of optical and UV spectroscopic observations, as well as a figure showing all of our optical spectra. In Appendix $\mathrm{C}$ we include more details about the atomic data used for our spectral modeling. In Appendix D we show the spectrum, line-flux measurements, and photometry that was used to derive the properties of the host galaxy.

\section{Appendix A UV and Optical Photometry}

Here we provide our optical and UV photometry (Table 5) and the blackbody fits to this photometry used to derive the photospheric evolution (Figure 23). 


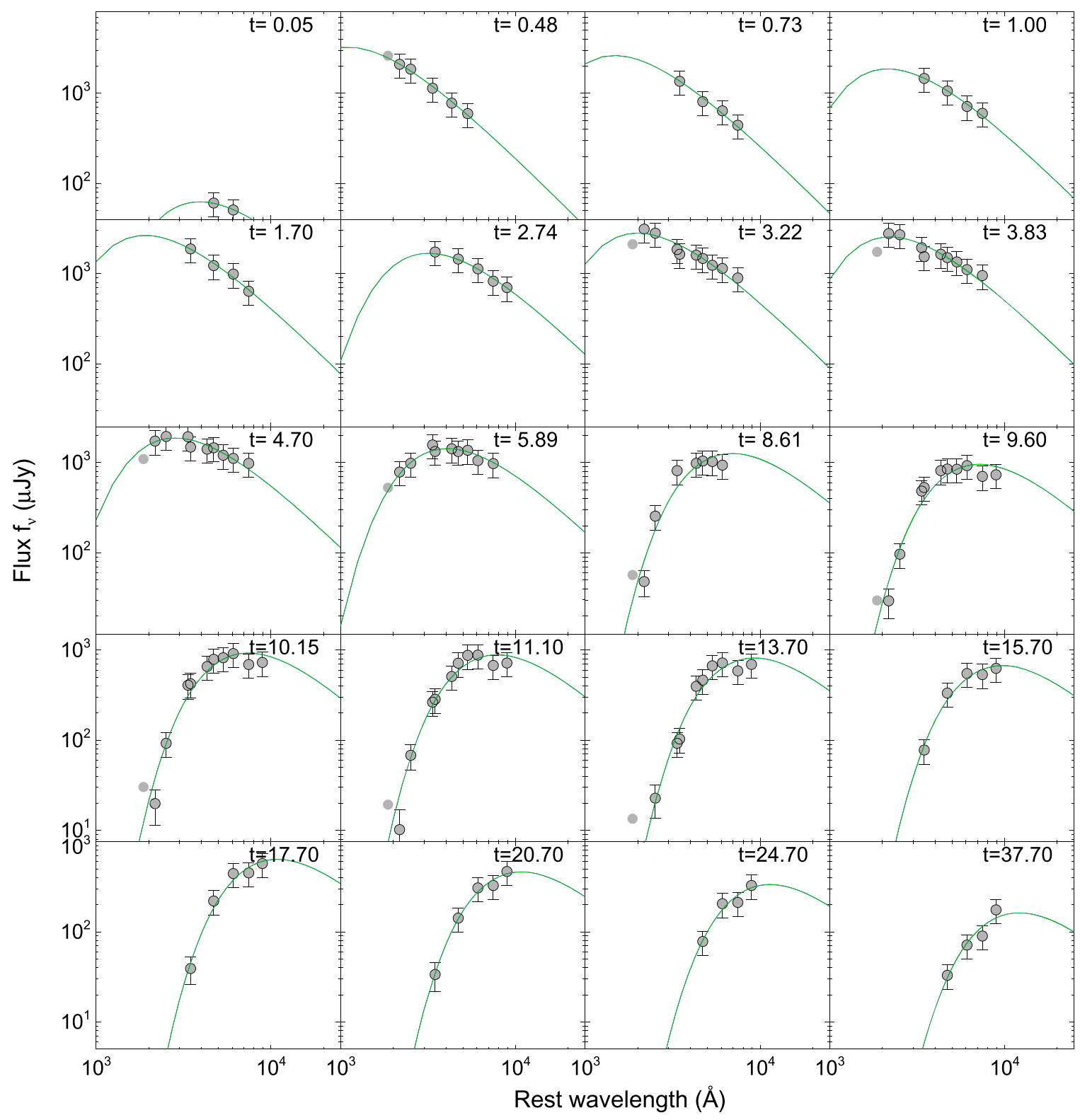

Figure 23. Blackbody fits to Swift/UVOT and optical photometry for SN2018gep. Since the UVOT and ground-based observations were taken at slightly different epochs, we interpolated the data in time using UVOT epochs at early times and LT epochs at later times.

Table 5

Optical and Ultraviolet Photometry for SN2018gep

\begin{tabular}{lcccc}
\hline \hline Date (JD) & $\Delta t$ & Instrument & Filter & Error in \\
AB Mag
\end{tabular}

Note. Table 5 is published in its entirety in the machine-readable format. A portion is shown here for guidance regarding its form and content.

(This table is available in its entirety in machine-readable form.) 


\section{Appendix B}

\section{UV and Optical Spectroscopy}

The observation log of our UV and optical spectra is provided in Table 6 . A plot showing the full sequence of optical spectrais shown in Figure 24.

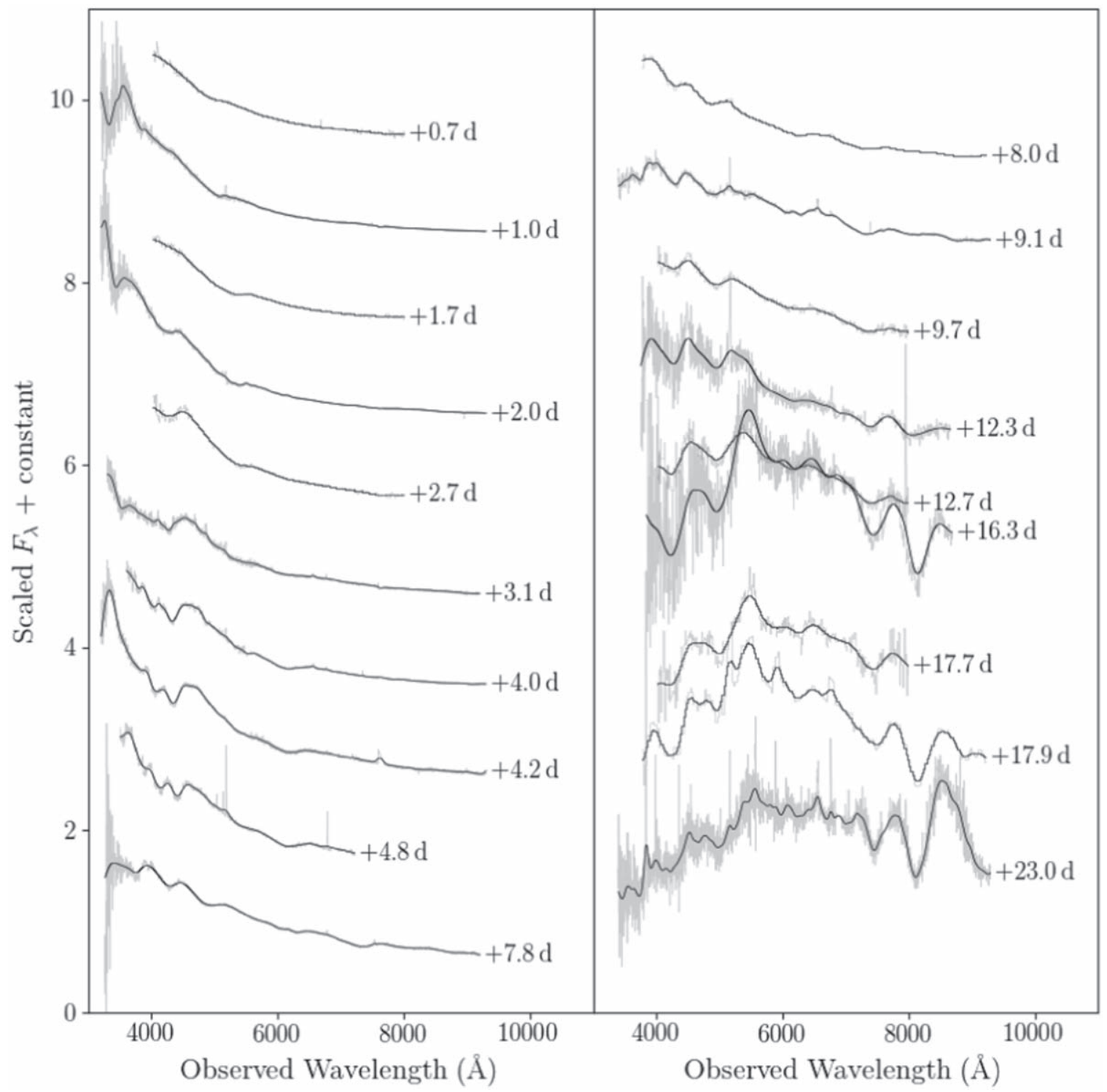

Figure 24. Ground-based optical spectra of SN2018gep. The light gray represents the observed spectrum, interpolating over host emission lines and telluric features. The black line is a Gaussian-smoothed version of the spectrum, using a Gaussian width that is several times the width of a galaxy emission line at that resolution. For more details on the smoothing procedure, see Section 2.1 of Ho et al. (2017). 
Table 6

Log of SN2018gep Optical Spectra

\begin{tabular}{|c|c|c|c|c|}
\hline Start Time (UTC) & $\Delta t$ & Instrument & Exp. Time (s) & Airmass \\
\hline 2018 Sep 09 20:30:01 & 0.7 & LT+SPRAT & 1200 & 1.107 \\
\hline 2018 Sep 10 04:28:51 & 1.0 & P200+DBSP & 600 & 1.283 \\
\hline 2018 Sep 10 21:03:42 & 1.7 & LT+SPRAT & 900 & 1.182 \\
\hline 2018 Sep 11 20:22:35 & 2.7 & LT+SPRAT & 900 & 1.107 \\
\hline 2018 Sep 12 06:09:59 & 3.1 & P200+DBSP & & \\
\hline 2018 Sep 13 03:52:58 & 4.0 & P200+DBSP & 300 & 1.209 \\
\hline 2018 Sep 17 04:38:40 & 8.0 & P60+SEDM & 1440 & 1.435 \\
\hline 2018 Sep 17 20:40:25.750 & 8.7 & $\mathrm{NOT}+\mathrm{ALFOSC}$ & 1800 & 1.19 \\
\hline 2018 Sep 18 05:21:58 & 9.1 & P200+DBSP & 600 & 1.720 \\
\hline 2018 Sep 18 20:14:35 & 9.7 & LT+SPRAT & 1000 & 1.143 \\
\hline 2018 Sep 21 11:15:10 & 12.3 & $\mathrm{XLT}+\mathrm{BFOSC}$ & 3000 & 1.181 \\
\hline 2018 Sep 21 20:58:21 & 12.7 & LT+SPRAT & 1000 & 1.293 \\
\hline 2018 Nov 09 05:26:17 & 61.1 & Keck I+LRIS & 900 & 3.242 \\
\hline
\end{tabular}

Note. Gratings used: Wasatch600 (LT+SPRAT), Gr4 (NOT+ALFOSC), 600/4000 (P200+DBSP; blue side), 316/7500 (P200+DBSP; red side), 400/8500 (Keck I

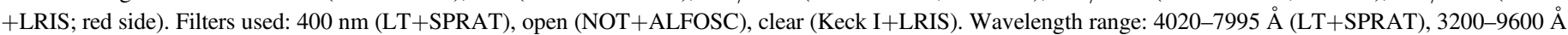
(NOT+ALFOSC), 1759-10311 Å (Keck I+LRIS), 3777-9223 ̊̊ (P60+SEDM). Resolution: 20 (LT+SPRAT), 710 (NOT+ALFOSC).

\section{Appendix C Atomic Data for Spectral Modeling}

The atomic data used for the spectral modeling in Section 3.2 is the same as described in Appendix A.4 of Ergon et al. (2018), but with the following modifications. The stage II-IV ions were (whenever possible) updated to include at least 50 levels for $\mathrm{N}, \mathrm{Na}, \mathrm{Al}, \mathrm{Ar}$, and $\mathrm{Ca}$, at least 100 levels for $\mathrm{C}, \mathrm{O}$, $\mathrm{Ne}, \mathrm{Mg}, \mathrm{Si}$, and $\mathrm{S}$, and at least 300 levels for $\mathrm{Sc}, \mathrm{Ti}, \mathrm{V}, \mathrm{Cr}, \mathrm{Mn}$, $\mathrm{Fe}, \mathrm{Co}$, and $\mathrm{Ni}$. In addition we updated the $\mathrm{C}$ II-C IV and $\mathrm{O}$ II$\mathrm{O}$ III ions with specific recombination rates from the online table by S. Nahar. ${ }^{42}$

\section{Appendix D \\ Data for Measuring Host Properties}

In this section we provide the data that we used to derive properties of the host galaxy of SN2018gep: the host-galaxy spectrum (Figure 25), line fluxes extracted from this spectrum (Table 7), and host-galaxy photometry (Table 8).

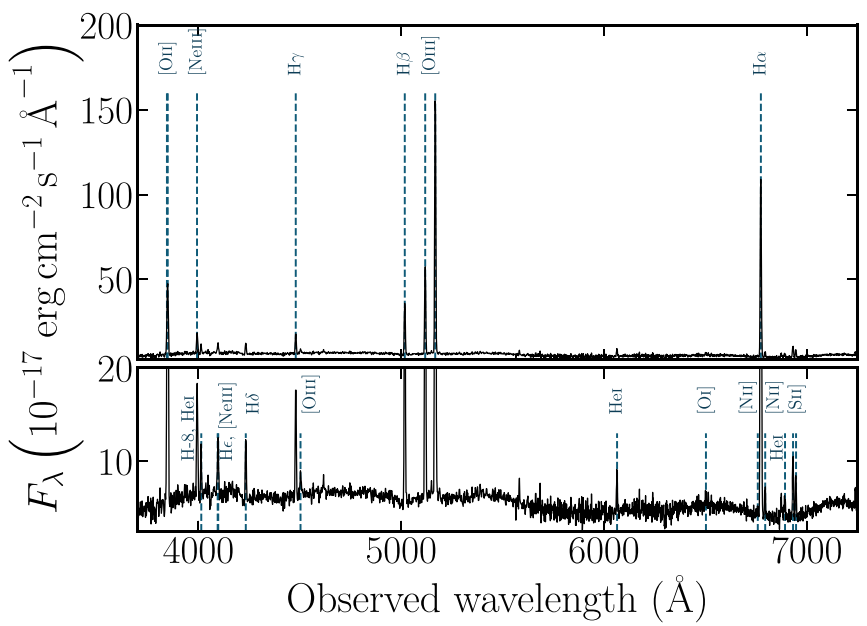

Figure 25. Host spectrum of SN2018gep obtained with Keck/LRIS on 2018 November 9, about two months after explosion. Strong emission lines from the host galaxy are labeled. The low host metallicity of 0.1 solar is reflected by very small $\mathrm{N}$ II $/ \mathrm{H} \alpha$ flux ratio. The large rest-frame [O III] $\lambda 5007$ equivalent width of $>160 \AA$ puts the host also in regime of extreme emission-line galaxies. This galaxy class constitutes $<2 \%$ of all star-forming galaxies at $z<0.3$ in the SDSS DR15 catalog. The undulations are due to the supernova. The spectrum is truncated at $7250 \AA$ for presentation purposes, and it is corrected for Galactic reddening.

$\overline{42}$ http://www.astronomy.ohio-state.edu/ nahar/_naharradiativeatomicdata/ 
Table 7

Line Fluxes from the Host Galaxy of SN2018gep Extracted from the Keck/LRIS Spectrum Obtained on 2018 November 9

\begin{tabular}{lcc}
\hline \hline Transition & $\begin{array}{c}\lambda_{\text {abs }} \\
(\mathrm{A})\end{array}$ & $\begin{array}{c}F \\
\left(10^{-17} \mathrm{erg} \mathrm{cm}^{-2} \mathrm{~s}^{-1}\right)\end{array}$ \\
\hline$[\mathrm{O}$ II] $\lambda \lambda 3726,3729$ & $3848.17 \pm 0.05$ & $334.5 \pm 6.23$ \\
{$[\mathrm{Ne}$ III] $\lambda 3869$} & $3993.50 \pm 0.16$ & $82.34 \pm 6.18$ \\
$\mathrm{He}$ I $\lambda 3889, \mathrm{H}-8$ & $4014.49 \pm 0.16$ & $29.01 \pm 4.73$ \\
{$[\mathrm{Ne}$ III] $\lambda 3968, \mathrm{H} \epsilon$} & $4096.66 \pm 0.26$ & $36.61 \pm 3.98$ \\
$\mathrm{H} \delta$ & $4233.87 \pm 0.13$ & $44.88 \pm 2.59$ \\
$\mathrm{H} \gamma$ & $4480.20 \pm 0.10$ & $81.95 \pm 3.74$ \\
{$[\mathrm{O}$ III] $\lambda 4364$} & $4503.68 \pm 0.10$ & $15.01 \pm 2.69$ \\
$\mathrm{H} \beta$ & $5017.87 \pm 0.08$ & $213.41 \pm 10.53$ \\
{$[\mathrm{O}$ III] $\lambda 4960$} & $5118.61 \pm 0.04$ & $352.42 \pm 6.50$ \\
{$[\mathrm{O}$ III] $\lambda 5008$} & $5168.04 \pm 0.04$ & $1066.70 \pm 19.50$ \\
$\mathrm{He}$ I $\lambda 5877$ & $6064.21 \pm 0.20$ & $27.04 \pm 2.30$ \\
$\mathrm{O}$ I $\lambda 6302$ & $6502.18 \pm 1.08$ & $6.72 \pm 2.94$ \\
{$[\mathrm{~N} \mathrm{II}] \lambda 6549$} & $6758.16 \pm 0.02$ & $11.15 \pm 6.73$ \\
$\mathrm{H} \alpha$ & $6773.40 \pm 0.02$ & $723.85 \pm 7.65$ \\
{$[\mathrm{~N} \mathrm{II}] \lambda 6585$} & $6794.67 \pm 0.02$ & $19.01 \pm 5.76$ \\
{$[\mathrm{He}$ I] $\lambda 6678$} & $6890.29 \pm 0.14$ & $7.88 \pm 2.19$ \\
{$[\mathrm{~S} \mathrm{II}] \lambda 6718$} & $6931.83 \pm 0.10$ & $41.76 \pm 2.38$ \\
{$[\mathrm{~S} \mathrm{II}] \lambda 6732$} & $6946.68 \pm 0.10$ & $28.15 \pm 2.19$
\end{tabular}

Note. All measurements are corrected for Galactic reddening.

Table 8

Brightness of the Host Galaxy from UV to IR Wavelenghts

\begin{tabular}{|c|c|c|c|c|c|}
\hline $\begin{array}{l}\text { Instrument/ } \\
\text { Filter }\end{array}$ & $\begin{array}{l}\lambda_{\text {eff }} \\
(\AA)\end{array}$ & $\begin{array}{l}\text { Brightness } \\
\quad \text { (mag) }\end{array}$ & $\begin{array}{l}\text { Instrument/ } \\
\text { Filter }\end{array}$ & $\begin{array}{l}\lambda_{\text {eff }} \\
(\AA)\end{array}$ & $\begin{array}{l}\text { Brightness } \\
\quad(\mathrm{mag})\end{array}$ \\
\hline GALEX/FUV & 1542.3 & $20.20 \pm 0.03$ & $\mathrm{SDSS} / i^{\prime}$ & 7439.5 & $18.62 \pm 0.04$ \\
\hline GALEX/NUV & 2274.4 & $20.09 \pm 0.03$ & $\mathrm{SDSS} / z^{\prime}$ & 8897.1 & $18.59 \pm 0.12$ \\
\hline $\mathrm{UVOT} / w 2$ & 2030.5 & $19.91 \pm 0.12$ & $\mathrm{PS} 1 / g_{\mathrm{PS} 1}$ & 4775.6 & $18.96 \pm 0.04$ \\
\hline $\mathrm{UVOT} / m 2$ & 2228.1 & $20.00 \pm 0.14$ & $\mathrm{PS} 1 / r_{\mathrm{PS} 1}$ & 6129.5 & $18.82 \pm 0.04$ \\
\hline $\mathrm{UVOT} / w 1$ & 2589.1 & $20.11 \pm 0.16$ & $\mathrm{PS} 1 / i_{\mathrm{PS} 1}$ & 7484.6 & $18.88 \pm 0.04$ \\
\hline $\mathrm{UVOT} / u$ & 3501.2 & $19.74 \pm 0.16$ & $\mathrm{PS} 1 / z_{\mathrm{PS} 1}$ & 8657.8 & $18.71 \pm 0.05$ \\
\hline UVOT $/ b$ & 4328.6 & $19.45 \pm 0.20$ & WIRCam $/ J$ & 12481.5 & $18.99 \pm 0.09$ \\
\hline $\mathrm{UVOT} / v$ & 5402.1 & $18.45 \pm 0.21$ & 2MASS $/ H$ & 16620.0 & $18.33 \pm 0.36$ \\
\hline $\mathrm{SDSS} / u^{\prime}$ & 3594.9 & $19.97 \pm 0.12$ & $W I S E / W 1$ & 33526.0 & $19.39 \pm 0.08$ \\
\hline $\mathrm{SDSS} / g^{\prime}$ & 4640.4 & $18.88 \pm 0.02$ & $W I S E / W 2$ & 46028.0 & $19.85 \pm 0.19$ \\
\hline $\mathrm{SDSS} / r^{\prime}$ & 6122.3 & $18.76 \pm 0.05$ & & & \\
\hline
\end{tabular}

Note. All measurements are reported in the AB system and are not corrected for reddening. For guidance, we report the effective wavelengths of each filter. 


\section{ORCID iDs}

Anna Y. Q. Ho (1D https://orcid.org/0000-0002-9017-3567 Daniel A. Goldstein (i) https://orcid.org/0000-0003-3461-8661 Steve Schulze (i) https://orcid.org/0000-0001-6797-1889 David K. Khatami (i) https://orcid.org/0000-0003-4307-0589 Daniel A. Perley (iD https://orcid.org/0000-0001-8472-1996 Avishay Gal-Yam (iD https://orcid.org/0000-0002-3653-5598 Alessandra Corsi (iD https://orcid.org/0000-0001-8104-3536 Igor Andreoni (iD https://orcid.org/0000-0002-8977-1498 Eric C. Bellm (ii) https://orcid.org/0000-0001-8018-5348 Nadia Blagorodnova (I) https://orcid.org/0000-00030901-1606

Joe S. Bright (iD https://orcid.org/0000-0002-7735-5796

S. Bradley Cenko (i) https://orcid.org/0000-0003-1673-970X Virginia Cunningham (1) https://orcid.org/0000-00032292-0441

Kishalay De (D) https://orcid.org/0000-0002-8989-0542 Claes Fransson (iD https://orcid.org/0000-0001-8532-3594 Christoffer Fremling (i) https://orcid.org/0000-00024223-103X

Adam Goldstein (iD https://orcid.org/0000-0002-0587-7042

Matthew J. Graham (i) https://orcid.org/0000-0002-3168-0139

Mansi M. Kasliwal (iD https://orcid.org/0000-0002-5619-4938

S. R. Kulkarni (i) https://orcid.org/0000-0001-5390-8563

Thomas Kupfer (iD https://orcid.org/0000-0002-6540-1484

Ragnhild Lunnan (iD https://orcid.org/0000-0001-9454-4639

Frank J. Masci (D) https://orcid.org/0000-0002-8532-9395

Chow-Choong Ngeow (i) https://orcid.org/0000-00018771-7554

Peter E. Nugent (1) https://orcid.org/0000-0002-3389-0586

Eran O. Ofek (D) https://orcid.org/0000-0002-6786-8774

Maria T. Patterson (ib https://orcid.org/0000-0002-4753-3387

Jesper Sollerman (ib https://orcid.org/0000-0003-1546-6615

Maayane T. Soumagnac (i) https://orcid.org/0000-00016753-1488

Xiaofeng Wang (iD https://orcid.org/0000-0002-7334-2357

Yuhan Yao (iD https://orcid.org/0000-0001-6747-8509

\section{References}

Ahn, C. P., Alexandroff, R., Allende Prieto, C., et al. 2012, ApJS, 203, 21 Ahn, C. P., Alexandroff, R., Allende Prieto, C., et al. 2014, ApJS, 211, 17 Alard, C., \& Lupton, R. H. 1998, ApJ, 503, 325

Almgren, A. S., Beckner, V. E., Bell, J. B., et al. 2010, ApJ, 715, 1221 Arcavi, I., Wolf, W. M., Howell, D. A., et al. 2016, ApJ, 819, 35 Arnouts, S., Cristiani, S., Moscardini, L., et al. 1999, MNRAS, 310, 540 Asplund, M., Grevesse, N., Sauval, A. J., \& Scott, P. 2009, ARA\&A, 47, 481 Astropy Collaboration, Price-Whelan, A. M., Sipőcz, B. M., et al. 2018, AJ, 156,123

Astropy Collaboration, Robitaille, T. P., Tollerud, J. E., et al. 2013, A\&A, 558, A33

Barbary, K. 2016, Extinction, v0.3.0, Zenodo, doi:10.5281/zenodo.804967 Barthelmy, S. D., Barbier, L. M., Cummings, J. R., et al. 2005, SSRv, 120, 143 Becker, A. 2015, HOTPANTS: High Order Transform of PSF and Template Subtraction, Astrophysics Source Code Library, ascl:1504.004

Bellm, E. C., Kulkarni, S.R., Barlow, T.R., et al. 2019b, PASP, 131, 1000 Bellm, E. C., Kulkarni, S. R., Graham, M. J., et al. 2019a, PASP, 131, 018002 Ben-Ami, S., Gal-Yam, A., Filippenko, A. V., et al. 2012, ApJL, 760, L33 Bersier, D., Fruchter, A. S., Strolger, L.-G., et al. 2006, ApJ, 643, 284 Bersten, M. C., Folatelli, G., García, F., et al. 2018, Natur, 554, 497 Bertin, E. 2010, SWarp: Resampling and Co-adding FITS Images Together, Astrophysics Source Code Library, ascl:1010.068

Blackburn, L., Briggs, M. S., Camp, J., et al. 2015, ApJS, 217, 8

Blagorodnova, N., Neill, J. D., Walters, R., et al. 2018, PASP, 130, 035003 Blanton, M. R., \& Roweis, S. 2007, AJ, 133, 734

Blaufuss, E. 2018, ATel, 12062, 1
Bourne, N., Maddox, S. J., Dunne, L., et al. 2012, MNRAS, 421, 3027 Branch, D., Benetti, S., Kasen, D., et al. 2002, ApJ, 566, 1005

Breeveld, A. A., Landsman, W., Holland, S. T., et al. 2011, AIP Conf. Ser., Gamma Ray Bursts 2010 (Melville, NY: AIP), 373

Bruzual, G., \& Charlot, S. 2003, MNRAS, 344, 1000

Bufano, F., Pian, E., Sollerman, J., et al. 2012, ApJ, 753, 67

Calzetti, D., Armus, L., Bohlin, R. C., et al. 2000, ApJ, 533, 682

Campana, S., Mangano, V., Blustin, A. J., et al. 2006, Natur, 442, 1008

Cano, Z. 2013, MNRAS, 434, 1098

Cano, Z., Izzo, L., de Ugarte Postigo, A., et al. 2017, A\&A, 605, A107

Cano, Z., Wang, S.-Q., Dai, Z.-G., \& Wu, X.-F. 2017, AdAst, 2017, 8929054

Cenko, S. B., Fox, D. B., Moon, D.-S., et al. 2006, PASP, 118, 1396 Chabrier, G. 2003, PASP, 115, 763

Chambers, K. C., Magnier, E. A., Metcalfe, N., et al. 2016, arXiv:1612.05560 Chevalier, R. A. 1998, ApJ, 499, 810

Chevalier, R. A., \& Irwin, C. M. 2011, ApJL, 729, L6

Corsi, A., Ofek, E. O., Gal-Yam, A., et al. 2012, ApJL, 747, L5

Corsi, A., Ofek, E. O., Gal-Yam, A., et al. 2014, ApJ, 782, 42

Costantin, L., Avramova-Bonche, A., Pinter, V., et al. 2018, ATel, 12047, 1

Cutri, R. M., Wright, E. L., \& Conrow, T. 2013, Explanatory Supplement to the AllWISE Data Release Products

De, K., Kasliwal, M. M., Ofek, E. O., et al. 2018, Sci, 362, 201

Dekany, R., Smith, R. M., Belicki, J., et al. 2016, Proc. SPIE, 9908, 99085M Dessart, L. 2019, A\&A, 621, A141

Drout, M. R., Chornock, R., Soderberg, A. M., et al. 2014, ApJ, 794, 23 Duev, D. A., Mahabal, A., Masci, F. J., et al. 2019, MNRAS, 489, 3582 Ergon, M., Fransson, C., Jerkstrand, A., et al. 2018, A\&A, 620, A156 Evans, P. A., Beardmore, A. P., Page, K. L., et al. 2009, MNRAS, 397, 1177 Fitzpatrick, E. L. 1999, PASP, 111, 63

Foley, R. J., Papenkova, M. S., Swift, B. J., et al. 2003, PASP, 115, 1220

Fremling, C., Sollerman, J., Taddia, F., et al. 2016, A\&A, 593, A68

Galama, T. J., Vreeswijk, P. M., van Paradijs, J., et al. 1998, Natur, 395, 670 Gal-Yam, A. 2019a, ApJ, 882, 102

Gal-Yam, A. 2019b, ARA\&A, 57, 305

Garmire, G. P., Bautz, M. W., Ford, P. G., Nousek, J. A., \& Ricker, G. R., Jr. 2003, Proc. SPIE, 4851, 28

Gehrels, N., Chincarini, G., Giommi, P., et al. 2004, ApJ, 611, 1005 Goldstein, A., Burns, E., Hamburg, R., et al. 2016, arXiv:1612.02395 Gorosabel, J., Pérez-Ramírez, D., Sollerman, J., et al. 2005, A\&A, 444, 711 Graham, M. J., Kulkarni, S. R., Bellm, E. C., et al. 2019, PASP, 131, 1001 Groh, J. H., Meynet, G., Georgy, C., \& Ekström, S. 2013, A\&A, 558, A131 Gruber, D., Goldstein, A., Weller von Ahlefeld, V., et al. 2014, ApJS, 211, 12 Gruen, D., Seitz, S., \& Bernstein, G. M. 2014, PASP, 126, 158

Han, X. H., Hammer, F., Liang, Y. C., et al. 2010, A\&A, 514, A24

Hickish, J., Razavi-Ghods, N., Perrott, Y. C., et al. 2018, MNRAS, 475, 5677

Hjorth, J., Malesani, D., Jakobsson, P., et al. 2012, ApJ, 756, 187

Ho, A. Y. Q., Ness, M. K., Hogg, D. W., et al. 2017, ApJ, 836, 5

Ho, A. Y. Q., Perley, D. A., Hallinan, G., et al. 2018b, ATel, 12056, 1

Ho, A. Y. Q., Phinney, E. S., Ravi, V., et al. 2019, ApJ, 871, 73

Ho, A. Y. Q., Schulze, S., Perley, D. A., et al. 2018c, ATel, 12030, 1

Ho, P. T. P., Moran, J. M., \& Lo, K. Y. 2004, ApJL, 616, L1

Högbom, J. A. 1974, A\&AS, 15, 417

Howell, D. A., Kasen, D., Lidman, C., et al. 2013, ApJ, 779, 98

Hudelot, P., Cuillandre, J.-C., Withington, K., et al. 2012, yCat, 2317, 0

Hunter, J. D. 2007, CSE, 9, 90

Ilbert, O., Arnouts, S., McCracken, H. J., et al. 2006, A\&A, 457, 841

Izotov, Y. I., Stasińska, G., Meynet, G., Guseva, N. G., \& Thuan, T. X. 2006, A\&A, 448, 955

Izzo, L., de Ugarte Postigo, A., Maeda, K., et al. 2019, Natur, 565, 324

Izzo, L., Thöne, C. C., Schulze, S., et al. 2017, MNRAS, 472, 4480 Jerkstrand, A., Ergon, M., Smartt, S. J., et al. 2015, A\&A, 573, A12

Jones, E., Oliphant, E., Peterson, P., et al. 2001, SciPy, http://www.scipy.org/ Kasen, D. 2017, in Handbook of Supernovae, ed. A. W. Alsabti \& P. Murdin (Cham: Springer Int. Publishing AG), 939

Kasen, D., \& Bildsten, L. 2010, ApJ, 717, 245

Kasen, D., Metzger, B. D., \& Bildsten, L. 2016, ApJ, 821, 36

Kasliwal, M. M., Cannella, C., Bagdasaryan, A., et al. 2019, PASP, 131, 038003

Katz, B., Kushnir, D., \& Dong, S. 2013, arXiv:1301.6766

Kauffmann, G., Heckman, T. M., Tremonti, C., et al. 2003, MNRAS, 346, 1055

Kennicutt, R. C., Jr. 1998, ARA\&A, 36, 189

Kewley, L. J., Dopita, M. A., Sutherland, R. S., et al. 2001, ApJ, 556, 121

Kocevski, D., Modjaz, M., Bloom, J. S., et al. 2007, ApJ, 663, 1180

Krühler, T., Malesani, D., Fynbo, J. P. U., et al. 2015, A\&A, 581, A125

Kuin, N. P. M., Wu, K., Oates, S., et al. 2019, MNRAS, 487, 2505 
Kuin, N. P. M., Landsman, W., Breeveld, A. A., et al. 2015, MNRAS, 449, 2514

Laskar, T., Coppejans, D. L., Margutti, R., \& Alexander, K. D. 2017, GCN, 22216, 1

Law, N. M., Kulkarni, S. R., Dekany, R. G., et al. 2009, PASP, 121, 1395

Levesque, E. M., Kewley, L. J., Graham, J. F., \& Fruchter, A. S. 2010, ApJL, 712, L26

Lupton, R., Blanton, M. R., Fekete, G., et al. 2004, PASP, 116, 133

Luridiana, V., Morisset, C., \& Shaw, R. A. 2015, A\&A, 573, A42

Lyman, J. D., Bersier, D., James, P. A., et al. 2016, MNRAS, 457, 328

Lyutikov, M., \& Toonen, S. 2019, MNRAS, 487, 5618

Madau, P., \& Dickinson, M. 2014, ARA\&A, 52, 415

Mahabal, A., Rebbapragada, U., Walters, R., et al. 2019, PASP, 131, 038002

Malesani, D., Tagliaferri, G., Chincarini, G., et al. 2004, ApJL, 609, L5

Mannucci, F., Cresci, G., Maiolino, R., Marconi, A., \& Gnerucci, A. 2010 MNRAS, 408, 2115

Margutti, R., Chincarini, G., Covino, S., et al. 2007, A\&A, 474, 815

Margutti, R., Metzger, B. D., Chornock, R., et al. 2019, ApJ, 872, 18

Marino, R. A., Rosales-Ortega, F. F., Sánchez, S. F., et al. 2013, A\&A, 559, A114

Martin, D. C., Fanson, J., Schiminovich, D., et al. 2005, ApJL, 619, L1

Masci, F. J., Laher, R. R., Rusholme, B., et al. 2019, PASP, 131, 018003

Matzner, C. D., \& McKee, C. F. 1999, ApJ, 510, 379

Mazzali, P. A., Sullivan, M., Pian, E., et al. 2016, MNRAS, 458, 3455

Mazzali, P. A., Valenti, S., Della Valle, M., et al. 2008, Sci, 321, 1185

McMullin, J. P., Waters, B., Schiebel, D., Young, W., \& Golap, K. 2007, adass XVI, 376, 127

Meegan, C., Lichti, G., Bhat, P. N., et al. 2009, ApJ, 702, 791

Michałowski, M. J., Hjorth, J., Malesani, D., et al. 2009, ApJ, 693, 347

Modjaz, M., Bianco, F. B., Siwek, M., et al. 2019, arXiv:1901.00872

Modjaz, M., Li, W., Butler, N., et al. 2009, ApJ, 702, 226

Modjaz, M., Liu, Y. Q., Bianco, F. B., \& Graur, O. 2016, ApJ, 832, 108

Moriya, T. J., Chen, T.-W., \& Langer, N. 2017, ApJ, 835, 177

Moriya, T. J., Sorokina, E. I., \& Chevalier, R. A. 2018, SSRv, 214, 59

Moriya, T. J., \& Tominaga, N. 2012, ApJ, 747, 118

Murphy, E. J., Condon, J. J., Schinnerer, E., et al. 2011, ApJ, 737, 67

Nakar, E., \& Piro, A. L. 2014, ApJ, 788, 193

Nakar, E., \& Sari, R. 2010, ApJ, 725, 904

Narayana Bhat, P., Meegan, C. A., von Kienlin, A., et al. 2016, ApJS, 223, 28

Nicholl, M., Smartt, S. J., Jerkstrand, A., et al. 2013, Natur, 502, 346

Oke, J. B., Cohen, J. G., Carr, M., et al. 1995, PASP, 107, 375

Oke, J. B., \& Gunn, J. E. 1982, PASP, 94, 586

Oliphant, T. E. 2006, A Guide to NumPy (Trelgol Publishing)

Osterbrock, D. E., \& Ferland, G. J. 2006, in Astrophysics of Gaseous Nebulae and Active Galactic Nuclei, II., ed. D. E. Osterbrock \& G. J. Ferland (Sausalito, CA: Univ. Science Books)

Ovaldsen, J.-E., Jaunsen, A. O., Fynbo, J. P. U., et al. 2007, ApJ, 662, 294

Patterson, M. T., Bellm, E. C., Rusholme, B., et al. 2019, PASP, 131, 018001

Pérez, F., \& Granger, B. E. 2007, CSE, 9, 21

Perley, D. A. 2019, PASP, 131, 1002

Perley, D. A., Mazzali, P. A., Yan, L., et al. 2019, MNRAS, 484, 1031
Perrott, Y. C., Scaife, A. M. M., Green, D. A., et al. 2013, MNRAS, 429, 3330 Pettini, M., \& Pagel, B. E. J. 2004, MNRAS, 348, L59

Piascik, A. S., Steele, I. A., Bates, S. D., et al. 2014, Proc. SPIE, 9147, 91478H Piro, A. L. 2015, ApJL, 808, L51

Planck Collaboration, Ade, P. A. R., Aghanim, N., et al. 2016, A\&A, 594, A13

Poznanski, D., Chornock, R., Nugent, P. E., et al. 2010, Sci, 327, 58

Prentice, S. J., Maguire, K., Smartt, S. J., et al. 2018, ApJL, 865, L3

Pursiainen, M., Childress, M., Smith, M., et al. 2018, MNRAS, 481, 894

Quimby, R. M., De Cia, A., Gal-Yam, A., et al. 2018, ApJ, 855, 2

Quimby, R. M., Kulkarni, S. R., Kasliwal, M. M., et al. 2011, Natur, 474, 487

Rest, A., Garnavich, P. M., Khatami, D., et al. 2018, NatAs, 2, 307

Rigault, M., Neill, J. D., Blagorodnova, N., et al. 2019, A\&A, 627, A115

Roming, P. W. A., Kennedy, T. E., Mason, K. O., et al. 2005, SSRv, 120 , 95

Sault, R. J., Teuben, P. J., \& Wright, M. C. H. 1995, adass IV, 77, 433

Schlafly, E. F., \& Finkbeiner, D. P. 2011, ApJ, 737, 103

Schulze, S., Ho, A. Y. Q., \& Mill, A. A. 2018a, ATel, 12032, 1

Schulze, S., Krühler, T., Leloudas, G., et al. 2018b, MNRAS, 473, 1258

Schulze, S., Malesani, D., Cucchiara, A., et al. 2014, A\&A, 566, A102

Shivvers, I., Zheng, W. K., Mauerhan, J., et al. 2016, MNRAS, 461, 3057

Skrutskie, M. F., Cutri, R. M., Stiening, R., et al. 2006, AJ, 131, 1163

Smartt, S. J., Clark, P., Smith, K. W., et al. 2018, ATel, 11727, 1

Smith, N. 2014, ARA\&A, 52, 487

Soderberg, A. M., Chakraborti, S., Pignata, G., et al. 2010, Natur, 463, 513

Stanway, E. R., Levan, A. J., Tanvir, N., et al. 2015, MNRAS, 446, 3911

Starling, R. L. C., Wiersema, K., Levan, A. J., et al. 2011, MNRAS, 411, 2792

Steele, I. A., Smith, R. J., Rees, P.-C., et al. 2004, Proc. SPIE, 5489, 679S

Tachibana, Y., \& Miller, A. A. 2018, PASP, 130, 128001

Taddia, F., Sollerman, J., Fremling, C., et al. 2019, A\&A, 621, A71

Tanaka, M., Tominaga, N., Morokuma, T., et al. 2016, ApJ, 819, 5

Thöne, C. C., Christensen, L., Prochaska, J. X., et al. 2014, MNRAS, 441, 2034

Thöne, C. C., Fynbo, J. P. U., Östlin, G., et al. 2008, ApJ, 676, 1151

Tonry, J. L., Denneau, L., Heinze, A. N., et al. 2018, PASP, 130, 064505

Toy, V. L., Cenko, S. B., Silverman, J. M., et al. 2016, ApJ, 818, 79

Van der Walt, S. J., Crellin-Quick, A., \& Bloom, J. S. 2019, JOSS, 4, 1247

von Kienlin, A., Meegan, C. A., Paciesas, W. S., et al. 2014, ApJS, 211, 13

Wang, J., Xin, L., Ren, J., et al. 2018, ATel, 12055, 1

Wang, L. J., Wang, X. F., Cano, Z., et al. 2017, MNRAS, 489, 1110

Whitesides, L., Lunnan, R., Kasliwal, M. M., et al. 2017, ApJ, 851, 107

Woosley, S. E. 2010, ApJL, 719, L204

Woosley, S. E., \& Heger, A. 2007, PhR, 442, 269

Wright, A. H., Robotham, A. S. G., Bourne, N., et al. 2016, MNRAS, 460, 765

Wright, E. L., Eisenhardt, P. R. M., Mainzer, A. K., et al. 2010, AJ, 140, 1868

Wygoda, N., Elbaz, Y., \& Katz, B. 2019, MNRAS, 484, 3941

Yan, L., Quimby, R., Gal-Yam, A., et al. 2017, ApJ, 840, 57

Yaron, O., \& Gal-Yam, A. 2012, PASP, 124, 668

Yaron, O., Perley, D. A., Gal-Yam, A., et al. 2017, NatPh, 13, 510

Zhang, W., Howell, L., Almgren, A., Burrows, A., \& Bell, J. 2011, ApJS, 196, 20

Zwart, J. T. L., Barker, R. W., Biddulph, P., et al. 2008, MNRAS, 391, 1545 